\title{
Estimation of a Joint Model for the Term Structure of Interest Rates and the Macroeconomy
}

\author{
Hans Dewachter ${ }^{a, b *}$, Marco Lyrio $^{a}$ and Konstantijn Maes $^{a}$ \\ ${ }^{a}$ CES, Catholic University of Leuven \\ ${ }^{b}$ RIFM, Erasmus University Rotterdam
}

First version: June 2001

This version: August 2001

\begin{abstract}
In this paper, we present a stylized continuous time model integrating the macroeconomy and the bond markets. We use this framework to estimate (real) interest rate policy rules using information contained in both macroeconomic variables (i.e. output and inflation) and in the term structure of interest rates. We extend the standard Kalman filter procedure in order to estimate this model efficiently. Application to the U.S. economy shows that this model is able to estimate the macroeconomic dynamics accurately and that the standard feedback rule only in observable factors is not valid within this framework. Moreover, we find that observable macroeconomic variables do not explain much of the term structure. However, (filtered) stochastic central tendencies of these macroeconomic variables do. Finally, both observable and non-observable factors determine the risk premia and hence the excess holding returns of the bonds.
\end{abstract}

Keywords: Essentially affine term structure model, feedback interest rate rule, forecasting.

J.E.L.: E43, E44, E52.

\footnotetext{
${ }^{*}$ Corresponding author. Details for correspondence: Center for Economic Studies, Naamsestraat 69, B-3000 Leuven, Belgium. Tel: (+)32 (0)16 326859, email: hans.dewachter@econ.kuleuven.ac.be. Konstantijn Maes is Aspirant of the FWO-Vlaanderen. We are grateful for financial support from the FWO-Vlaanderen (Project No.: G.0332.01). We thank seminar participants at the University of Frankfurt and, in particular, Yunus Aksoy, Frank Smets and Volcker Wieland for useful comments. Obviously they do not share any responsibility for remaining errors.
} 


\section{Introduction}

In this paper, we present and estimate a continuous time term structure model incorporating both observable economic aggregates (output and inflation) and latent variables. This model is thus capable of describing formally the interrelations between the macroeconomy and the bond markets. Such a formal description is important for several reasons. First, it allows us to take the standard (multi-factor) term structure models one level deeper by at least partially linking and interpreting the latent factors in terms of observable economic aggregates. Second, it makes possible the estimation and validation of policy reaction functions of a central banker (the so-called feedback rules) based on information in the entire term structure (instead of information contained in the short rate only). Third, it enables the analysis of the effects of alternative monetary policy rules on the bond markets throughout the spectrum of maturities. In this paper, we mainly focus on the first two reasons for introducing this new model and estimation technique.

Turning to the first of the reasons mentioned, one motivation to estimate such a hybrid model is the (partial) identification of the driving factors behind the yield curve. Standard (multi-factor) term structure literature identifies the determinants of the dynamics of the yield curve mainly in terms of factors defined on the yield curve itself. These factors are then frequently labeled as level, slope and curvature factors according to their effects on the yield curve. However, these concepts do not bring us any further in understanding the driving forces behind the yield curve. An alternative to these fully latent factors is the derivation of a complete general equilibrium model linking the term structure to the exogenous factors of the economy (see, for instance, Bakshi and Chen (1996), Berardi (2001), Buraschi (1996) and $\mathrm{Wu}(2000))$. This approach has the advantage that the latent factors are defined on the whole economy and thus have a clear and unambiguous meaning in terms of economic aggregates. However, given the current state of explicit general equilibrium modeling, it may be too early to impose all of the cross-sectional restrictions imposed by any such a general equilibrium model on the empirical data. In this paper, we follow the alternative (intermediate) route of identifying the driving forces behind the term structure. More specifically, we only impose the necessary no-arbitrage conditions on a continuous time vector autoregressive (VAR) system containing both observable and latent factors. As such, we avoid the need to fully specify the structure of the economy while still retaining the possibility of a (partial) identification of the driving factors behind the term structure in terms of observable macroeconomic aggregates (see Ang and Piazzesi (2001), Piazzesi (2001) and Fleming and Remolona (2001)). ${ }^{1}$ The contribution of this paper is that we recast these VAR models in a continuous time framework

\footnotetext{
${ }^{1}$ Obviously, the inclusion of observable factors does not necessarily imply identification of the latent factors in the model. However, we allow for a clear and unambiguous identification of the latent factors by construction. Specifically, we impose the latent factors to serve as stochastic attractors for the observable factors.
} 
that allows for an unambiguous interpretation of the latent factors. More specifically, the model is such that latent factors have the interpretation of central tendencies, i.e. long-run expectations of the observable (macro) factors. As shown by Kozicki and Tinsley (2001), modeling long-run expectations (endpoints in their terminology) is crucial for fitting the longer end of the yield curve.

The second reason to adopt the procedure presented in this paper is related to the determination of the equilibrium instantaneous riskless interest rate. While general equilibrium models can endogenously derive the equilibrium interest rate, other approaches cannot. In order to obtain the dynamics of the instantaneous interest rate, it has become standard to assume a direct link between monetary policy and this instantaneous rate in the form of a feedback interest rate rule (see, for instance, Wu (2000) or Ang and Piazzesi (1999)). Over the past ten years, these policy rules have been studied extensively with mixed success. For instance, in a multi-country study Clarida, Galí and Gertler (1998) find mixed evidence for the existence of a Taylor rule. The Taylor rule seems to be successful in replicating the dynamics of the short-run (say, three months) interest rates, especially after 1980. However, it fails entirely in modeling the entire term structure dynamics. More specifically, when estimating Taylor rules in an arbitrage-free model for the term structure, we find that the Taylor rule breaks down completely! This suggests that some crucial factors modeling the dynamics of the longer end of the yield curve are left out in this standard Taylor rule (see also Kozicki and Tinsley (2001)). These factors are included in this paper in terms of latent factors. We also define the equilibrium instantaneous interest rate in terms of an error-correction model (ECM) with a long-run attractor comprised of a long-run policy rule for the real interest rate. This policy rule is defined in terms of both observable and non-observable macroeconomic factors. This definition of the instantaneous interest rate closes the model. Together with the no-arbitrage conditions and assumptions about the prices of risk, the interest rate definition determines the sensitivities of other bond rates and, as such, determines the whole term structure of interest rates. In this way, we contribute to the literature by estimating policy rules on the entire term structure of interest rates, as opposed to the standard approach of estimating Taylor rules on short- term interest rates only. Our model also provides a panel data test on the validity of feedback policy rules. If monetary authorities follow some type of feedback rule known by the market then the dynamics of the variables entering the policy rule together with the (assumed) risk attitude of agents determine the entire term structure of interest rates in a complete capital market.

Finally, our approach, i.e. casting the model in continuous time, gives us several advantages over the existing literature. First, only by estimating a continuous time model, we can analytically impose the no-arbitrage condition carefully within a broad class of models. As such, arbitrage opportunities can be ruled out in continuous time and hence also for any 
longer discrete time investment interval. Second, although we face the standard Vasicek restrictions for the dynamics of the factors, we extend the model to allow for time-varying risk premia along the lines of Duffee (2001) and Dai and Singleton (2001). This extension allows us to analyze the effects of macroeconomic variables on the size and sign of the risk premia. Third, our model identifies structural shocks in both the observable and latent variables and thus allows us to analyze impulse responses and variance decompositions of the yield curve in terms of well-defined structural shocks.

The remainder of the paper is organized as follows. In Section 2, we discuss the macroeconomic model we impose on the data. This model is expressed in terms of the VAR dynamics both in terms of the output gap and inflation as well as in terms of the effects of latent factors. We also introduce the continuous time (real) interest rate policy rule along the lines stated above. Subsequently, we derive the bond price determination by stating the continuous time macro model in discrete time state space form. Section 3 deals with the empirical implementation of the model. A crucial problem is that the introduction of observable factors (containing strong cyclical components, for instance in the output gap) may generate imaginary eigenvalues, rendering the traditional spectral decomposition estimation procedure of term structure models, as proposed in the literature (e.g. de Jong (2000)), theoretically infeasible ${ }^{2}$. We introduce an alternative estimation procedure that solves the basic problem -the computation of a matrix exponential- in an efficient way. The estimation results are presented in Section 4. In this section we also perform a variance decomposition analysis in order to measure the importance of macroeconomic shocks in the variability of the yield curve. Next, we analyze the impulse responses of the yield curve to the different structural shocks and we end the section with an evaluation of the forecasting performance of the model. We conclude in Section 5 by summarizing the main findings and pointing out some issues for future research.

\section{$2 \quad$ A stylized model}

The model is constructed in such a way that it fits perfectly within the class of affine term structure models (ATSM). As such, it is well-suited for analyzing the implications of observable macroeconomic aggregates for the term structure of interest rates. First, we set out the assumptions concerning the macroeconomic framework and the monetary policy rule. Second, we rewrite the model in a general state space representation and, finally, we analyze the implications for the term structure of interest rates.

\footnotetext{
${ }^{2}$ According to Dai and Singleton (1997, NBER working paper version of Dai and Singleton, 2000), "the assumption that the eigenvalues are real rules out some potentially interesting dynamics associated with complex eigenvalues". Beaglehole and Tenney (1991) expand the class of interest rate processes to allow more dynamic possibilities. In particular, they present processes with decaying oscillatory behavior.
} 


\subsection{Dynamics of macroeconomic and latent factors}

In this section, we present a simple and stylized continuous time model for the dynamics of macroeconomic aggregates, i.e. the output gap $y(t)$ and inflation $\pi(t)$. In order to ease the empirical implementation of the model, we assume from the start that backward-looking models are good approximations of reality. The macroeconomic model is then built by the assumptions imposed on its dynamics:

$$
\begin{aligned}
& \mathrm{d} y(t)=\left[\kappa_{y y}\left(y^{*}(t)-y(t)\right)+\kappa_{y \pi}\left(\pi^{*}(t)-\pi(t)\right)+\kappa_{y \rho}\left(\rho^{*}(t)-\rho(t)\right)\right] d t+\sigma_{y} d W_{y}(t) \\
& \mathrm{d} \pi(t)=\left[\kappa_{\pi y}\left(y^{*}(t)-y(t)\right)+\kappa_{\pi \pi}\left(\pi^{*}(t)-\pi(t)\right)+\kappa_{\pi \rho}\left(\rho^{*}(t)-\rho(t)\right)\right] d t+\sigma_{\pi} d W_{\pi}(t) \\
& \mathrm{d} y^{*}(t)=\kappa_{y^{*} y^{*}}\left(\theta_{y^{*}}-y^{*}(t)\right) \mathrm{d} t+\sigma_{y^{*}} d W_{y^{*}}(t) \\
& \mathrm{d} \pi^{*}(t)=\kappa_{\pi^{*} \pi^{*}}\left(\theta_{\pi^{*}}-\pi^{*}(t)\right) \mathrm{d} t+\sigma_{\pi^{*}} d W_{\pi^{*}}(t)
\end{aligned}
$$

where $W_{i}(t), i=\left\{y, \pi, y^{*}, \pi^{*}\right\}$, denote independent Wiener processes defined on the probability space $(\Omega, \mathcal{F}, P)$ with filtration $\mathcal{F}_{t}$. As such, we can interpret the shocks $d W(t)$ as structural shocks in output gap, inflation and structural (long-run) output gap and inflation, respectively. The dynamics of the system are basically modeled in terms of (latent) central tendencies $\left(y^{*}\right.$ and $\left.\pi^{*}\right)$ and in terms of deviations from these central tendencies, e.g. $\left(y(t)-y^{*}(t)\right)$. Formally, we only allow deviations from the central tendencies to determine the short-run dynamics of the respective macroeconomic variables. In this way, we actually ensure that the exogenous central tendency variables will act as long-run attractors in this system. ${ }^{3}$ The model is closed with the following definition for the instantaneous interest rate $r(t)$ :

$$
r(t) \equiv \pi(t)+\rho(t)
$$

We (implicitly) assume that the monetary authority uses a feedback rule for the real interest rate. More specifically, we assume that changes in the (ex-post) real interest rate $\rho$ are a response to deviations of the output gap and/or inflation from their central tendencies and to a mean reverting (real interest rate smoothing) component relative to a stochastic long run mean $\rho^{*}(t)$ :

$$
\begin{aligned}
& \mathrm{d} \rho(t)=\left(\kappa_{\rho y}\left(y^{*}(t)-y(t)\right)+\kappa_{\rho \pi}\left(\pi^{*}(t)-\pi(t)\right)+\kappa_{\rho \rho}\left(\rho^{*}(t)-\rho(t)\right)\right) \mathrm{d} t+\sigma_{\rho} \mathrm{d} W_{\rho}(t) \\
& \rho^{*}(t)=\gamma_{0}+\gamma_{y} y(t)+\gamma_{\pi} \pi(t)+\gamma_{y^{*}} y^{*}(t)+\gamma_{\pi^{*}} \pi^{*}(t) .
\end{aligned}
$$

\footnotetext{
${ }^{3}$ Note that these long-run central tendencies can only serve as long-run attractors if the dynamics are stable. In the estimation of the system we impose stability of the factors and thus the long-run attracting property of the exogenous central tendencies.
} 
Note that the above equations define a central bank policy rule in terms of the real interest rate dynamics. This policy rule can be decomposed into a long-run policy rule for the real interest rate, captured by the stochastic process $\rho^{*}(t)$. We allow this long run rule to be dependent on both observed macro-economic series as well as their central tendencies. However, The central bank also has a short run policy function, as can be inferred from the actual expected dynamics in (3). The central bank also responds to deviations in the output and inflation from their central tendencies.

The dynamics conform well to the standard macroeconomic view. More specifically, we allow each of the observable economic variables, output gap and inflation, to be affected through three channels: the (instantaneous) real interest rate $(\rho)$, the other economic variable (output gap or inflation) and, finally, a mean reverting component modeling the possible inertia in the adjustment process. Central tendencies of output and inflation are assumed to be strictly exogenous and independent processes.

The above representation of the dynamics of the economy can easily be restated in matrix notation. Denoting $n$ as the number of factors in the model, five in our case, we define the vectors of $n$ factors and shocks and an $n \times n$ diagonal matrix $\mathbf{S}$ as:

$$
\begin{aligned}
& \mathbf{f}(t) \equiv\left(\begin{array}{l}
y(t) \\
\pi(t) \\
\rho(t) \\
y^{*}(t) \\
\pi^{*}(t)
\end{array}\right), \quad \mathrm{d} \mathbf{W}(t) \equiv\left(\begin{array}{l}
\mathrm{d} W_{y}(t) \\
\mathrm{d} W_{\pi}(t) \\
\mathrm{d} W_{\rho}(t) \\
\mathrm{d} W_{y^{*}}(t) \\
\mathrm{d} W_{\pi^{*}}(t)
\end{array}\right), \text { and } \\
& \mathbf{S} \equiv \operatorname{diag}\left(\sigma_{y}, \sigma_{\pi}, \sigma_{\rho}, \sigma_{y^{*}}, \sigma_{\pi^{*}}\right)
\end{aligned}
$$

the dynamics of the economy can be restated as follows:

$$
\mathrm{d} \mathbf{f}(t)=\mathbf{K}(\boldsymbol{\psi}-\mathbf{f}(t)) \mathrm{d} t+\mathbf{S} \mathrm{d} \mathbf{W}(t),
$$

where

$$
\mathbf{K}=\left[\begin{array}{ccccc}
\kappa_{y y}-\kappa_{y \rho} \gamma_{y} & \kappa_{y \pi}-\kappa_{y \rho} \gamma_{\pi} & \kappa_{y \rho} & -\kappa_{y y}-\kappa_{y \rho} \gamma_{y^{*}} & -\kappa_{y \pi}-\kappa_{y \rho} \gamma_{\pi^{*}} \\
\kappa_{\pi y}-\kappa_{\pi \rho} \gamma_{y} & \kappa_{\pi \pi}-\kappa_{\pi \rho} \gamma_{\pi} & \kappa_{\pi \rho} & -\kappa_{\pi y}-\kappa_{\pi \rho} \gamma_{y^{*}} & -\kappa_{\pi \pi}-\kappa_{\pi \rho} \gamma_{\pi^{*}} \\
\kappa_{\rho y}-\kappa_{\rho \rho} \gamma_{y} & \kappa_{\rho \pi}-\kappa_{\rho \rho} \gamma_{\pi} & \kappa_{\rho \rho} & -\kappa_{\rho y}-\kappa_{\rho \rho} \gamma_{y^{*}} & -\kappa_{\rho \pi}-\kappa_{\rho \rho} \gamma_{\pi^{*}} \\
0 & 0 & 0 & \kappa_{y^{*} y^{*}} & 0 \\
0 & 0 & 0 & 0 & \kappa_{\pi^{*} \pi^{*}}
\end{array}\right]
$$

and

$$
\boldsymbol{\psi}=\mathbf{K}^{-1}\left(\kappa_{y \rho} \gamma_{0}, \kappa_{\pi \rho} \gamma_{0}, \kappa_{\rho \rho} \gamma_{0}, \kappa_{y^{*} y^{*}} \theta_{y^{*}}, \kappa_{\pi^{*} \pi^{*}} \theta_{\pi^{*}}\right)^{\prime} .
$$

Note that due to the fact that the matrix $\mathbf{K}$ is in general not diagonal, closed form equations for the expectation of the level and/or the covariance matrix of the factors are not easily obtained. Obviously, these concepts are of great importance in terms of forecasting the future evolution of the state (of the economy). Running ahead of things, one can construct 
the mean and covariance matrix of the factors by numerically solving a system of ordinary differential equations (ODEs) for the raw moments of each of the factors involved (see Section 3.1.2 below).

\subsection{Implications for bond markets}

Equation (5) completely specifies the dynamics of the macroeconomic variables and the instantaneous (policy) interest rate. This system, therefore, must also determine (up to some risk premium component) the term structure of interest rates and its dynamics. Absence of arbitrage opportunities in fact implies that zero-coupon default-free bond prices at time $t$, maturing at $T, p(t, T)$ are defined as:

$$
p(t, T)=E_{t}^{Q}\left(\exp \left(-\int_{t}^{T} r(u) d u\right),\right.
$$

where $Q$ denotes the risk-neutral probability measure. In general, this risk-neutral probability is unknown and can only be specified by assuming some specification for the prices of factor risk. Following Duffee (2001), time variability in the prices of risk can be captured by specifying prices of risk as an affine function of the latent factors. The vector containing the (time-varying) prices of risk $\boldsymbol{\xi}$ is defined as:

$$
\boldsymbol{\xi}(t)=\mathbf{S} \boldsymbol{\Lambda}+\mathbf{S}^{-1} \boldsymbol{\Xi} \mathbf{f}(t)
$$

where $\boldsymbol{\Lambda} \equiv\left(\lambda_{y}, \lambda_{\pi}, \lambda_{\rho}, \lambda_{y^{*}} \lambda_{\pi^{*}}\right)^{\prime}$ and $\boldsymbol{\Xi}$ an $n \times \mathrm{x} n$ matrix containing the sensitivities of the prices of risk to the levels of the state space factors. Changing measures is then easily done by means of the Girsanov theorem:

$$
\mathrm{d} \mathbf{W}(t)=\mathrm{d} \tilde{\mathbf{W}}(t)-\boldsymbol{\xi}(t) \mathrm{d} t
$$

where $\tilde{W}_{i}(t)$ constitutes a martingale under measure $Q$. State space dynamics can be restated in terms of this risk-neutral metric $Q$ as:

$$
\begin{aligned}
& \mathrm{d} \mathbf{f}(t)=\tilde{\mathbf{K}}(\tilde{\boldsymbol{\psi}}-\mathbf{f}(t)) \mathrm{d} t+\mathbf{S} \mathrm{d} \tilde{\mathbf{W}}(t) \\
& \tilde{\mathbf{K}}=\mathbf{K}+\boldsymbol{\Xi} \\
& \tilde{\boldsymbol{\psi}}=\tilde{\mathbf{K}}^{-1}\left(\mathbf{K} \boldsymbol{\psi}-\mathbf{S}^{2} \boldsymbol{\Lambda}\right)
\end{aligned}
$$

A functional form for bond prices can be obtained by assuming that bond prices are time homogeneous functions of the factors $\mathbf{f}(t)$ and the time to maturity $\tau \equiv T-t$ :

$$
p(t, T)=p(\mathbf{f}(t), \tau)=\exp \left(-a(\tau)-\mathbf{b}(\tau)^{\prime} \mathbf{f}(t)\right)
$$


where $\mathbf{b}(\tau)$ is an $n \times 1$ vector and by imposing the no-arbitrage condition in the bond markets:

$$
\mathcal{D}^{Q}(p(\mathbf{f}(t), \tau))=r(t) p(\mathbf{f}(t), \tau)
$$

where $\mathcal{D}^{Q}$ denotes the Dynkin operator under the probability measure $Q$. The intuitive meaning of the latter condition is that, once transformed to a risk-neutral world, instantaneous holding returns for all bonds are equal to the instantaneous riskless interest rate. Obviously, using Girsanov's theorem we can immediately infer the implications for the real world by changing measure from the risk-neutral one to the historical measure $P$. Changing measure only affects the drift and, therefore, the instantaneous holding return under the historical probability measure for a bond with maturity $\tau$ may be written as:

$$
\frac{\mathcal{D}^{P}(p(\mathbf{f}(t), \tau))}{p(\mathbf{f}(t), \tau)}=r(t)-\mathbf{b}(\tau)^{\prime} \mathbf{S} \boldsymbol{\xi}(t)
$$

Equations (9) and (10) determine the solution for the functions $a(\tau)$ and $\mathbf{b}(\tau)$ in terms of a system of coupled ODEs that, in the general case, can only be solved numerically:

$$
\begin{aligned}
& \frac{\partial a(\tau)}{\partial \tau}=a_{0}+(\tilde{\mathbf{K}} \tilde{\boldsymbol{\psi}})^{\prime} \mathbf{b}(\tau)-\frac{1}{2} \sum_{i=1}^{n} b_{i}^{2}(\tau) S_{i i}^{2} \\
& \frac{\partial \mathbf{b}(\tau)}{\partial \tau}=\mathbf{b}_{0}-\tilde{\mathbf{K}}^{\prime} \mathbf{b}(\tau)
\end{aligned}
$$

A particular solution to this system of ODEs is obtained by specifying a set of initial conditions on $a$ and $\mathbf{b}$. Inspection of equation (9) immediately shows that the relevant initial conditions are: $a(0)=0$ and $\mathbf{b}(0)=\mathbf{0}$. The vectors of constants $a_{0}$ and $\mathbf{b}_{0}$ are defined by the interest rate definition equation (2) and take in the setting of this paper the values: $a_{0}=0$ and $\mathbf{b}_{0}$ $=\left(\begin{array}{lllll}0 & 1 & 1 & 0 & 0\end{array}\right)^{\prime}$.

The bond pricing solution differs in important ways from the standard (independent) multi-factor term structure literature. First, allowing for interrelations among the factors (i.e. non-zero off-diagonal elements in $\tilde{\mathbf{K}}$ ) generates a coupled system of ODEs instead of a set of uncoupled ODEs. The bond pricing solution for the $a$ and $\mathbf{b}$ functions, therefore, do not reduce to the standard multi-factor result (see, for instance, de Jong (2000)). Second, the factor loadings no longer start from unity at maturity $\tau=0$. The introduction of stochastic central tendencies makes that all of the these factors have zero loadings in the determination of the short rate. They only influence the instantaneous rate indirectly by serving as a long-run (stochastic) attractor. 


\section{Empirical implementation}

\subsection{Macroeconomic model in state space notation}

In this section, we present an efficient estimation method to estimate both the state-space dynamics, including observable and non-observable factors, as well as the prices of risk, implied by the term structure. The assumption of the presence of unobserved factors implies that some filtering procedure needs to be applied to recover the time series of the factors. In order to avoid ad hoc yield curve inversion procedures (Pearson and Sun (1994), Chen and Scott (1993)) we opt for a Kalman filter algorithm ${ }^{4}$. While the Kalman filter estimation procedure for affine models is well established in the case where all factors are assumed to be unobserved (see de Jong (2000) and Duan and Simonato (1999)), some issues remain to be settled once we incorporate observable factors into the state space vector, as is done in this paper. These issues can be decomposed into two classes. First, there is the issue of the inclusion of macroeconomic aggregates in the state space that raises two separate problems: (i) the possibility of imaginary eigenvalues with respect to the spectral decomposition of the matrix $\mathbf{K}$ in equation (5), and (ii) the issue of correctly updating the state vector $\mathbf{f}$. Second, there is the concern for efficiency in the estimation since conditional expectations and variance-covariance matrices are in general no longer available. We tackle these issues in the following sections.

\subsubsection{Measurement equation}

The model is tested on a data set containing yields of different maturities, output gap and inflation data. In order to estimate the parameters of this model, it is necessary to link the model to these variables via the measurement equation. First, with respect to the yield curve, we estimate the parameters so as to fit the observed yield curve as well as possible given the observed state vector. Let $\hat{\mathbf{y}}(t)$ denote the vector of yields observed at time $t$ for maturities $\tau_{i}, i=\{1, \ldots, m\}, \hat{\mathbf{y}}(t)=\left(\hat{y}_{1}\left(t, \tau_{1}\right), \ldots, \hat{y}_{m}\left(t, \tau_{m}\right)\right)^{\prime}$, where each $\hat{y}_{i}\left(t, \tau_{i}\right)$ is defined as $\hat{y}_{i}\left(t, \tau_{i}\right) \equiv-\frac{\ln \left(p\left(t, \tau_{i}\right)\right)}{\tau_{i}}$. Second, we use the implied one-step ahead predictions for the output gap $y$ and the inflation $\pi$ to fit the macroeconomic part of the model. Bringing these elements together, we require that the model's parameter estimates are optimized over the joint set of moments to be fit. That is, we define the measurement equation as:

\footnotetext{
${ }^{4}$ This Kalman filter procedure is less time consuming than other algorithms like the Simulated Method of Moments (SMM) technique. For our Gaussian model a linear Kalman filter together with exact maximum likelihood (ML) estimation is optimal within the class of all linear estimators. Parameter estimators can be shown to be efficient and consistent (see Bollerslev and Wooldridge (1992) for a proof). There is, however, one subtlety to be mentioned. Some of the factors are latent and based upon a linear prediction. These predicted state variables will enter in the conditional mean and variance imputing errors in the likelihood function. This complication, however, does not invalidate the asymptotic properties mentioned above. Finally, Duffee and Stanton (2000) advocate the use of the linear Kalman filter above the EMM and SNP auxiliary model estimation approach (see Gallant and Tauchen, 1992).
} 


$$
\left(\begin{array}{c}
\hat{y}_{1}\left(t, \tau_{1}\right) \\
\vdots \\
\hat{y}_{m}\left(t, \tau_{m}\right) \\
y(t) \\
\pi(t)
\end{array}\right)=\left(\begin{array}{c}
\mathbf{a} \\
0 \\
0
\end{array}\right)+\left(\begin{array}{c}
\mathbf{B} \\
\mathbf{e}_{1}^{\prime} \\
\mathbf{e}_{2}^{\prime}
\end{array}\right)\left(\begin{array}{c}
y(t) \\
\pi(t) \\
\rho(t) \\
y^{*}(t) \\
\pi^{*}(t)
\end{array}\right)+\boldsymbol{\varepsilon}_{t}
$$

where $\mathbf{e}_{i}$ is a $(n \times 1)$ column vector of zeros with a one on the $i$ th row, $\boldsymbol{\varepsilon}_{t}$ is an $(m+2) \times 1$ vector of measurement errors and

$$
\begin{aligned}
& \mathbf{a}=\left(a\left(\tau_{1}\right) / \tau_{1}, \ldots, a\left(\tau_{m}\right) / \tau_{m}\right)^{\prime} \\
& \mathbf{B}=\left(\begin{array}{c}
\mathbf{b}\left(\tau_{1}\right)^{\prime} / \tau_{1} \\
\vdots \\
\mathbf{b}\left(\tau_{n}\right)^{\prime} / \tau_{m}
\end{array}\right) .
\end{aligned}
$$

We can rewrite the measurement equation more concisely as:

$$
\begin{aligned}
& \mathbf{z}(t)=\mathbf{c}_{z}+\mathbf{H f}(t)+\boldsymbol{\varepsilon}(t) \\
& E_{t}\left(\boldsymbol{\varepsilon}(t) \boldsymbol{\varepsilon}^{\prime}(t)\right)=\mathbf{R},
\end{aligned}
$$

where $\mathbf{z}(t)$ denotes the LHS of (13).

\subsubsection{Transition equation}

It is standard in the term structure literature to transform the state space such that the $\mathbf{K}$ matrix in (5) becomes diagonal. This procedure yields well-known closed form solutions for the conditional expectations and conditional covariance matrix of the transformed factors (de Jong (2000)). Implicit in this approach is the assumption that the eigenvalues of $\mathbf{K}$ are all real. Denoting the transformed state factor $\mathbf{f}_{\mathrm{T}}(t) \equiv \mathbf{V} \mathbf{f}(t)$, where $\mathbf{V}$ is the matrix of eigenvectors of $\mathbf{K}: \mathbf{K}=\mathbf{V}^{-1} \mathbf{L V}$, the state space dynamics (5) can be restated as:

$$
\mathrm{d} \mathbf{f}_{\mathrm{T}}(t)=\mathbf{L}\left(\boldsymbol{\psi}_{\mathrm{T}}-\mathbf{f}_{\mathrm{T}}(t)\right) \mathrm{d} t+\mathbf{V S} \mathrm{d} \mathbf{W}(t),
$$

where $\boldsymbol{\psi}_{\mathrm{T}}$ denotes $\mathbf{V} \boldsymbol{\psi}$.

By taking this as the initial starting point, the term structure literature basically estimates the eigenvalues $\mathbf{L}$ and eigenvectors $\mathbf{V}$ of the system. This effectively implies that all eigenvalues (and accompanying eigenvectors) are by construction real. While this may be a reasonable assumption in the case of latent factors, it is no longer once macroeconomic aggregates are to be included in the state space. The latter variables possess some dynamic properties that cannot be ignored in the estimation (imaginary eigenvalues). As such, transforming the state space is no longer innocuous and can, therefore, not be done without possible major implications for the dynamics of the system. 
The conditional expectation of the level and the conditional covariance matrix of the factors are not easily obtained and require solving a matrix exponential. In Appendix A, it is shown that this can be done by solving the following equation:

$$
\frac{\mathrm{d} \mathbf{g}(t+h)}{\mathrm{d} h}=\Theta+\boldsymbol{\Omega} \mathbf{g}(t+h)
$$

where $h$ denotes the prediction horizon (one quarter in our case) and $\mathbf{g}(t+h)$ an $q \times 1$ vector of all possible first and second (raw) time $t$ conditional moments that can be constructed from the state vector $\mathbf{f}(t)$. The $q \times 1$ vector $\Theta$ and the $q \times q$ matrix $\boldsymbol{\Omega}$ are defined in Appendix A. One can rely on numerical procedures to solve the resulting ODEs, which in turn can be used to construct the conditional expectation and covariance matrix of the state vector.

We thus refrain from transforming the system in terms of eigenvalues and eigenvectors, implying that the closed form solution for expectations and covariance matrices that have been derived under this transformation are no longer available. The above argument thus basically restricts the space of state vectors to the one that contains the non-transformed macro-aggregates. Once the state vector is identified, the corresponding transition equation in the Kalman filter procedure for the time interval $h=\Delta t$ is also fixed to:

$$
\begin{aligned}
& \mathbf{f}(t+\Delta t)=\mathbf{c}+\boldsymbol{\Phi}(\Delta t)(\mathbf{f}(t)-\mathbf{c})+\mathbf{v}_{t+\Delta t} \\
& E\left(\mathbf{v}_{t+\Delta t} \mathbf{v}_{t+\Delta t}^{\prime}\right)=\mathbf{Q}(\Delta t) .
\end{aligned}
$$

While in principle the Kalman filter could be applied now, this is in practice infeasible since equation (18), computing the conditional expectation and covariance matrix, depends on the state vector through the initial value condition for the ODEs from (17). This dependence of the initial conditions on the state vector obliges us to solve for each time period the system of ODEs and renders estimation on reasonable sample sizes infeasible. In order to make the filter operationally efficient we impose stationarity on the state space dynamics which allows us to characterize the solution of (17) over any discrete time interval $\Delta t$ as a first order Markov process on the state space generated by the vector $\mathbf{g}$ :

$$
\mathbf{g}(t+\Delta t)=-\boldsymbol{\Omega}^{-\mathbf{1}} \Theta+\boldsymbol{\Gamma}(\Delta t)\left(\mathbf{g}(t)+\boldsymbol{\Omega}^{-\mathbf{1}} \Theta\right)
$$

Making $\mathbf{x}(t)=\mathbf{g}(t)+\boldsymbol{\Omega}^{-\mathbf{1}} \Theta$, we get

$$
\mathbf{x}(t+\Delta t)=\mathbf{\Gamma}(\Delta t) \mathbf{x}(t) .
$$

The $q$ x $q$ matrix $\boldsymbol{\Gamma}(\Delta t)$ can be recovered by:

$$
\boldsymbol{\Gamma}(\Delta t)=X(t+\Delta t)^{\prime} X(t)\left(X(t)^{\prime} X(t)\right)^{-1},
$$


where $X(t)$ denotes an $q \times \mathrm{x} q$ matrix stacking $q$ linearly independent starting vectors $\mathbf{x}(0)^{\prime}$ and $X(t+\Delta t)$ contains the stacked vectors of conditional expectations at $t+\Delta t\left(\mathbf{x}(t+\Delta t)^{\prime}\right)$ corresponding to each of the $q$ initial condition vectors. One can easily reconstruct the matrices $\boldsymbol{\Phi}(\Delta t)$ and $\mathbf{Q}(\Delta t)$ from the $\boldsymbol{\Gamma}(\Delta t)$ matrix. Note that this matrix is no longer time dependent but only depends on the parameters. This thus requires only a single computation of the $\boldsymbol{\Gamma}(\Delta t)$ matrix per function evaluation and, therefore, speeds up the estimation process considerably.

\subsection{The Kalman filter algorithm}

\subsubsection{Prediction equations}

From the transition equation above, we can see that $\mathbf{f}(t+\Delta t)$ is a linear combination of two random variables $\mathbf{f}(t)$ and $\mathbf{v}(t+\Delta t$ ), both (multivariate) normally distributed. Hence, $\mathbf{f}(t+\Delta t)$ is itself (multivariate) normal with mean and covariance matrix :

$$
\begin{aligned}
\hat{\mathbf{f}}_{t+\Delta t \mid t} & =\mathbf{c}+\boldsymbol{\Phi}\left(\hat{\mathbf{f}}_{t \mid t}-\mathbf{c}\right) \\
\hat{\mathbf{P}}_{t+\Delta t \mid t} & =\boldsymbol{\Phi} \hat{\mathbf{P}}_{t \mid t} \boldsymbol{\Phi}^{\prime}+\mathbf{Q}(\Delta t)
\end{aligned}
$$

\subsubsection{Updating equations}

The next step is to improve the precision of our estimator by taking into account the additional information in the update step. By applying a simple lemma (for a proof see Harvey (1989), pp. 165-166), we can show that the updated state vector (conditional on the $t+\Delta t$ observation of the data) is again multivariate normally distributed with mean and covariance matrix equal to:

$$
\begin{aligned}
& \hat{\mathbf{f}}_{t+\Delta t \mid t+\Delta t}=\hat{\mathbf{f}}_{t+\Delta t \mid t}+\hat{\mathbf{P}}_{t+\Delta t \mid t} \mathbf{H}^{\prime}\left(\mathbf{H} \hat{\mathbf{P}}_{t+\Delta t \mid t} \mathbf{H}^{\prime}+\mathbf{R}\right)^{-1}\left(\mathbf{z}(t+\Delta t)-\mathbf{c}_{z}-\mathbf{H} \hat{\mathbf{f}}_{t+\Delta t \mid t}\right) \\
& \hat{\mathbf{P}}_{t+\Delta t \mid t+\Delta t}=\hat{\mathbf{P}}_{t+\Delta t \mid t}-\hat{\mathbf{P}}_{t+\Delta t \mid t} \mathbf{H}^{\prime}\left(\mathbf{H} \hat{\mathbf{P}}_{t+\Delta t \mid t} \mathbf{H}^{\prime}+\mathbf{R}\right)^{-1} \mathbf{H} \hat{\mathbf{P}}_{t+\Delta t \mid t} .
\end{aligned}
$$

Note that this brings us to the second issue of correctly updating the state vector $\mathbf{f}$, which can be shown to be accomplished by assuming no measurement errors in the output and inflation series: i.e.: $\varepsilon_{m+1, t}=\varepsilon_{m+2, t}=0$.

\subsubsection{Prediction error decomposition and ML estimation}

Finally, the optimal filtering procedure is obtained by maximizing the loglikelihood with respect to the parameters describing the state space dynamics and the prices of factor risk, 


$$
\begin{aligned}
& \ell(\mathcal{Z} \mid \boldsymbol{\xi})=\sum_{t=1}^{n d}-\frac{1}{2} \ln \left|\mathbf{H} \hat{\mathbf{P}}_{t+\Delta t \mid t} \mathbf{H}^{\prime}+\mathbf{R}\right| \\
& -\sum_{t=1}^{n d} \frac{1}{2}\left(\mathbf{z}(t+\Delta t)-\mathbf{c}_{z}-\mathbf{H} \hat{\mathbf{f}}_{t+\Delta t \mid t}\right)^{\prime}\left(\mathbf{H} \hat{\mathbf{P}}_{t+\Delta t \mid t} \mathbf{H}^{\prime}+\mathbf{R}\right)^{-1}\left(\mathbf{z}(t+\Delta t)-\mathbf{c}_{z}-\mathbf{H} \hat{\mathbf{f}}_{t+\Delta t \mid t}\right) .
\end{aligned}
$$

where $n d$ stands for the number of observations in the data set.

\section{Estimation results}

\subsection{Data}

We base our analysis on spliced data from McCulloch and Kwon (1993) and Bliss (1997) provided by Duffee (2001). This data set consists of month-end yields on zero-coupon U.S. Treasury bonds with maturities of 3 and 6 months and 1,2, 5, and 10 years. We use a quarterly frequency in the construction of the time series in order to incorporate the output gap and inflation series. Our data set consists, therefore, of 164 data points (1958:Q1 to 1998:Q4) for each of the series. A proxy for the output gap is obtained by using a HodrickPrescott (HP) filter on the GDP series over the sample period. ${ }^{5}$ Inflation was constructed by taking the yearly percentage change in the CPI index, that is $\pi_{t}=\ln C P I_{t}-\ln C P I_{t-4}$. Figure (1) depicts the series for the output gap, inflation and the term structure.

\section{Insert Figure 1}

In Table 1 we give some descriptive statistics of the sample series. The average term structure series display an increasing yield curve and the observed variance of the term structure tends to decrease in the maturity. There is strong evidence against normality in most series in terms of skewness and excess kurtosis (both decreasing with maturity) and in terms of a summary Jarque-Bera statistic ( $p$-values are reported in the table). Also, strong autocorrelation is observed in all series over the sample period. Most interestingly, however, is the correlation matrix showing extreme correlation among the various bonds and significant but more moderate correlations between bonds on the one side and output gap or inflation on the other side. The strong correlation between bonds (decreasing with the maturity difference) suggests the presence of a few important factors driving the yield curve. While these factors may also be part of the set of driving processes in the output gap and in inflation, the lower degree of correlation suggests that the links between these macro-aggregates and the yield

\footnotetext{
${ }^{5}$ We use a standard "lambda" in the filtering procedure equal to 1600.
} 
curve is significantly smaller. The output gap and inflation are positively correlated with each other.

Table 1: Summary statistics for the data used (1964:Q2-1997:Q4)

\begin{tabular}{ccccccccc}
\hline \hline & yield $_{1 q}$ & yield $_{2 q}$ & yield $_{1 y r}$ & yield $_{2 y r}$ & yield $_{5 y r}$ & yield $_{10 y r}$ & y & $\boldsymbol{\pi}$ \\
\hline \hline Mean (\%) & 5.975 & 6.230 & 6.450 & 6.689 & 7.010 & 7.210 & -0.006 & 4.286 \\
Std. (\%) & 2.779 & 2.811 & 2.785 & 2.712 & 2.602 & 2.543 & 1.633 & 2.894 \\
Min (\%) & 0.739 & 0.915 & 1.303 & 1.903 & 2.554 & 2.916 & -4.689 & 0.473 \\
Max (\%) & 15.241 & 15.924 & 15.911 & 16.107 & 15.696 & 15.065 & 3.879 & 13.502 \\
Auto & 0.987 & 0.987 & 0.990 & 0.992 & 0.996 & 0.998 & 0.861 & 0.992 \\
Skew & 1.147 & 1.142 & 1.030 & 0.985 & 0.911 & 0.752 & -0.204 & 1.231 \\
& $(0.000)$ & $(0.000)$ & $(0.000)$ & $(0.000)$ & $(0.000)$ & $(0.000)$ & $(0.287)$ & $(0.000)$ \\
Kurt & 4.509 & 4.531 & 4.143 & 3.975 & 3.691 & 3.276 & 3.230 & 3.977 \\
& $(0.000)$ & $(0.000)$ & $(0.003)$ & $(0.011)$ & $(0.071)$ & $(0.470)$ & $(0.548)$ & $(0.011)$ \\
JB & 51.525 & 51.672 & 37.923 & 33.021 & 25.925 & 15.972 & 1.496 & 47.934 \\
& $(0.000)$ & $(0.000)$ & $(0.000)$ & $(0.000)$ & $(0.000)$ & $(0.000)$ & $(0.473)$ & $(0.000)$ \\
\hline \hline & & & & & & & & \\
\hline \hline yield $_{1 q}$ & 1.000 & & & & & & & \\
yield $_{2 q}$ & 0.996 & 1.000 & & & & & & \\
yield $_{1 y r}$ & 0.986 & 0.995 & 1.000 & & & & & \\
yield $_{2 y r}$ & 0.968 & 0.978 & 0.992 & 1.000 & & & & \\
yield $_{5 y r}$ & 0.923 & 0.934 & 0.957 & 0.984 & 1.000 & & & \\
yield $_{10 y r}$ & 0.887 & 0.896 & 0.923 & 0.960 & 0.993 & 1.000 & & \\
$\quad$ y & 0.199 & 0.193 & 0.168 & 0.105 & 0.020 & -0.021 & 1.000 & \\
$\pi_{\pi}$ & 0.728 & 0.729 & 0.714 & 0.682 & 0.640 & 0.623 & 0.083 & 1.000 \\
\hline \hline
\end{tabular}

The bond yield data are based on spliced data from McCulloch and Kwon (1993) and Bliss (1997) provided by Duffee (2001) and concern U.S. Treasury bonds with maturities of 3 and 6 months and 1, 2, 5, and 10 years. Output gap $(y)$ and inflation $(\pi)$ data are constructed as mentioned in the text. The data series cover the period from 1958:Q1 until 1998:Q4, totalling 164 quarterly time series observations. Mean denotes the sample arithmetic average, expressed as p.a. percentage, Std standard deviation, Min minimum, Max maximum, Auto the first order quarterly autocorrelation, Skew and Kurt stand for skewness and kurtosis, respectively, while underneath these statistics are the significance levels at which the null of no skewness and the null of no excess kurtosis may be rejected. $J B$ stands for the Jarque-Bera normality test statistic with the significance level at which the null of normality may be rejected underneath it.

\subsection{The failure of standard Taylor rules}

The correlations in Table 1 suggest that the modeling of the joint behavior of the yield curve and the output gap and inflation is relevant. Given the strong focus on Taylor rules in the current literature, these rules form a natural starting point in modeling the interrelations between the yield curve and the macroeconomy. Obviously, Taylor rules are only designed to fit the short-term policy rate and not (necessarily) the entire term structure. However, by consequence of the expectation hypothesis, if Taylor rules do determine the dynamics of the policy rate, yields of any maturity will to a large extent be determined by the dynamics 
imposed by the rule as well. ${ }^{6}$

\section{Insert Figure 2}

To analyze the performance of Taylor rules in fitting the entire yield curve, we estimate the short-term Taylor rule on the U.S. data described above and then project the rule to the longer maturities (a formal treatment can be found in Ang and Piazzesi (2000)). The fit of the Taylor rule across maturities can be found in Figure 2. Inspecting this figure, it is clear that the Taylor rule is not performing well in describing the whole term structure. The rule fits relatively well the short end of the yield curve, say up to one-year interest rates. However, going beyond this maturity one clearly observes a significant deterioration in the fit of the yields. Similar results have been reported by Kozicki and Tinsley (2001). The failure of standard Taylor rules in fitting yields with maturities beyond one year can be attributed either to time-varying risk premia or to the fact that some factors, exerting most of their influence in the long run, have been excluded from the rule. In the next subsection we estimate the model proposed in Section 2, containing both additional factors in the Taylor rule (in the form of central tendencies) as well as time-varying risk premia. The addition of these two factors proves to be crucial for the fitting of the long-term interest rate.

\subsection{Estimates and discussion}

In this subsection, we turn to the estimation results of the model. The full model was estimated in a single step procedure. Optimization was performed using the Broyden-FletcherGoldfarb-Shanno (BFGS) algorithm with a convergence tolerance for the gradient of the estimated coefficients equal to 0.0001. We checked the robustness of the 'optimum' reported by checking convergence from an array of starting points. ${ }^{7}$ The estimation results can be found in Table 2 .

The model was also estimated for the subperiods 1958:Q1 to 1979:Q2 (pre-Volcker) and 1982:Q1 to 1998:Q4. The results can be found in Appendix B. Although the estimates for these subperiods are somewhat different from the ones obtained for the whole sample period, the main qualitative results do not depend on the subperiod.

\footnotetext{
${ }^{6}$ In theory, risk premia will also play their role which becomes increasingly important the longer the maturity. However, it has been argued forcefully in the literature that expectations and not risk premia dominate the yield curve and, as such, one should not overestimate the role of these risk premia.

${ }^{7}$ Note that, given the large amount of parameters to be estimated, identification and checking of the 'optimum' is a painstaking operation. During our experiments, we found this 'optimum' to be rather stable. However, due to the intrinsic nonlinearities in the optimisation problem, there is no guarantee that the values reported actually correspond to a global maximum.
} 
Table 2: Maximum likelihood estimates (1958:Q1-1998:Q4)

\begin{tabular}{|c|c|c|c|c|c|c|}
\hline & $\mathbf{y}$ & $\pi$ & $\rho$ & $\mathbf{y}^{*}$ & $\pi^{*}$ & \\
\hline $\boldsymbol{\kappa}_{y,}$ & $\begin{array}{c}0.9984 \\
(0.1318)\end{array}$ & $\begin{array}{c}0.3313 \\
(0.1483)\end{array}$ & $\begin{array}{l}-0.1677 \\
(0.1251)\end{array}$ & & & \\
\hline $\boldsymbol{\kappa}_{\pi,}$ & $\begin{array}{l}-0.9231 \\
(0.1114)\end{array}$ & $\begin{array}{c}0.3898 \\
(0.1584)\end{array}$ & $\begin{array}{c}0.2454 \\
(0.1442)\end{array}$ & & & \\
\hline $\boldsymbol{\kappa}_{\rho,}$ & $\begin{array}{c}-0.0078 \\
(0.1318)\end{array}$ & $\begin{array}{c}3.3615 \\
(1.3385)\end{array}$ & $\begin{array}{c}3.5793 \\
(1.0488)\end{array}$ & & & \\
\hline $\boldsymbol{\kappa}_{y^{*}}$ & & & & $\begin{array}{c}0.4305 \\
(0.0508)\end{array}$ & & \\
\hline $\boldsymbol{\kappa}_{\pi^{*}}$ & & & & & $\begin{array}{c}0.0082 \\
(0.0026) \\
\end{array}$ & \\
\hline$\theta$. & & & & $\begin{array}{l}-0.00006 \\
(0.00065)\end{array}$ & $\begin{array}{c}0.14398 \\
(0.06249)\end{array}$ & \\
\hline$\gamma_{0}$ & & & $\begin{array}{c}0.00005 \\
(0.00074)\end{array}$ & & & \\
\hline$\gamma$ & $\begin{array}{l}-0.0395 \\
(0.1678)\end{array}$ & $\begin{array}{c}0.0698 \\
(0.1528)\end{array}$ & & $\begin{array}{c}-0.1691 \\
(2.6139)\end{array}$ & $\begin{array}{c}0.0395 \\
(0.1460)\end{array}$ & \\
\hline$\lambda$ & $\begin{array}{l}-23.6286 \\
(62.2786)\end{array}$ & $\begin{array}{c}-8.7690 \\
(255.0090)\end{array}$ & $\begin{array}{l}-17.3618 \\
(85.0428)\end{array}$ & & & \\
\hline$\Xi_{\rho,}$ & $\begin{array}{l}-6.3176 \\
(1.7927)\end{array}$ & $\begin{array}{l}12.4734 \\
(3.7758)\end{array}$ & $\begin{array}{l}14.2254 \\
(3.8115)\end{array}$ & $\begin{array}{c}240.4993 \\
(255.2660)\end{array}$ & $\begin{array}{l}-20.6599 \\
(6.1457)\end{array}$ & \\
\hline$\sigma^{2}$ & $\begin{array}{c}0.000352 \\
(0.000048)\end{array}$ & $\begin{array}{c}0.000111 \\
(0.000017)\end{array}$ & $\begin{array}{c}0.003182 \\
(0.001315)\end{array}$ & $\begin{array}{c}0.000003 \\
(0.000006)\end{array}$ & $\begin{array}{c}0.000057 \\
(0.000012)\end{array}$ & \\
\hline$\overline{\mathbf{R}_{1 q}}$ & 11.9558 & & & & & \\
\hline $\mathbf{R}_{2 q}$ & 10.6958 & 9.9042 & & & & \\
\hline $\mathbf{R}_{1 y r}$ & 3.7498 & 4.0094 & 2.4713 & & & \\
\hline $\mathbf{R}_{2 y r}$ & 0.0564 & 0.0002 & 0.0001 & 0.0441 & & \\
\hline $\mathbf{R}_{5 y r}$ & 0.2861 & 0.5109 & 0.0001 & 0.6240 & 2.2596 & \\
\hline $\mathbf{R}_{10 y r}$ & 0.0963 & 0.0763 & 0.0071 & 0.1527 & 0.7036 & 0.0007 \\
\hline
\end{tabular}

ML estimates with robust standard errors underneath. Only the lower diagonal of the measurement error covariance matrix is given. These values are multiplied by $10^{6}$. Total loglikelihood amounts to 6994.8216 or 42.6513 on average (excluding constant in the loglikelihood). 


\subsubsection{Dynamics and interpretation of the factors}

First we turn to the modeling of the macroeconomic variables. Figure 3 presents the filtered time series for the five factors involved: three observable ones (output gap, $y$; inflation, $\pi$; and real interest rate, $\rho$ ) and two non-observable ones (output gap and inflation central tendencies, $y^{*}$ and $\pi^{*}$, respectively). The time series "STrule" also presented in this figure is discussed below. Visual inspection shows that even though the central tendencies are basically latent factors, they capture rather well the long-run behavior of the observable series. Statistical analysis confirms the attracting properties of the central tendencies for the three observable factors (see Table 2 where the first three diagonal elements of the matrix $\mathbf{K}$ are significantly different from zero). Most of the estimated interaction terms (off-diagonal elements in $K$ ) are statistically significant at a $10 \%$ level. The estimation results indicate that output responds negatively to a temporary excess inflation (i.e. inflation in excess of the steady state equilibrium inflation rate, $\pi^{*}-\pi<0$ ) while it responds positively to a temporary excess real interest rate (although this result is not statistically significant). Inflation responses are more in line with economic intuition. First, a temporary demand shock (i.e. output in excess of the equilibrium capacity, $y^{*}-y<0$ ) tends to induce additional inflationary pressure while an excessively high real interest rate tends to reduce the inflationary pressure. Finally, the real interest rate tends to react primarily to inflation. More specifically, expectations of increasing inflation (i.e. $\pi^{*}-\pi>0$ ) tend to result in quite substantial increases in the real interest rate.

\section{Insert Figure 3}

The resulting fit of the three macroeconomic series is presented in Figure 4. The 'data' for the real interest rate was computed based on the three-month yield and is, therefore, an approximation of the instantaneous real interest rate. We consider the fit of these series as satisfactory.

\section{Insert Figure 4}

Table 3 presents some diagnostic statistics. We find that the state space dynamics are stable, i.e. real parts of the eigenvalues of the matrix $\mathbf{K}$ are positive. Moreover, note that $\mathbf{K}$ has imaginary eigenvalues, indicating an oscillating impulse response for the output gap and inflation. These series present a halving time of 1.25 years, possibly linked to the business cycle frequency. The instantaneous real interest rate presents a much lower halving time of about 2 months. The central tendency of output gap has a halving time of 1.6 years in contrast to the central tendency of inflation which presents a halving time of about 84 years, indicating that this type of shock is very inert. 
Table 3: Diagnostic statistics of the estimated model

\begin{tabular}{|c|c|c|c|c|c|c|}
\hline \hline & \multicolumn{2}{|c|}{ Eigenvalue } & Half time (yr) & \multicolumn{2}{|c|}{ Mean (\%) } & rp (\%) \\
& real & imag. & & data & emp. & emp. \\
\hline \hline$y$ & 0.555 & 0.511 & 1.25 & -0.06 & -0.02 & 0.83 \\
$\pi$ & 0.555 & -0.511 & 1.25 & 4.28 & 4.36 & 0.10 \\
$\rho$ & 3.833 & & 0.18 & 1.69 & 1.78 & \\
$y^{*}$ & 0.430 & & 1.61 & & 0.01 & \\
$\pi^{*}$ & 0.008 & & 84.28 & & 4.28 & \\
yield $_{1 q}$ & & & & 5.97 & 6.13 & 1.82 \\
yield $_{2 q}$ & & & & 6.23 & 6.39 & 1.92 \\
yield $_{1 y r}$ & & & & 6.45 & 6.61 & 2.05 \\
yield $_{2 y r}$ & & & & 6.69 & 6.85 & 2.18 \\
yield $_{5 y r}$ & & & & 7.00 & 7.16 & 2.18 \\
yield $_{10 y r}$ & & & & 7.21 & 7.35 & 2.14 \\
\hline \hline
\end{tabular}

Eigenvalues denote the eigenvalues of the $\mathbf{K}$ matrix, half time is computed based on the real part of each eigenvalue, emp denotes the result where the filtered factor means are used, $r p$ stands for risk premium on an annual basis.

Table 3 also presents some additional statistics concerning the yield curve fit. The average yield curve based on the fitted factors presents a good fit to the empirical average yield curve. The implied risk premia take somewhat higher values than recorded in the latent factor literature. We find risk premia of about two percent, increasing in the maturity and with a slight decrease at the long end of the term structure.

Based on the above results we can discuss the role and interpretation of the latent factors in the model. As mentioned before, given the significant positive coefficients $\kappa_{y y}, \kappa_{\pi \pi}$ and $\kappa_{\rho \rho}$, latent factors serve as an attractor to the observable macroeconomic series (i.e. output gap and inflation). As such, the latent factors can be seen as some kind of long-run forecast for the observable series. The eigenvalues presented in Table 3 for $y, \pi$ and $\rho$ may be used to obtain a more precise idea about the long-run forecasts. These eigenvalues indicate the speed of convergence of the observable macroeconomic variables to their central tendencies. Based on these numbers we find that for the output gap and inflation we have after five years approximately $93 \%$ and after ten years more than $99 \%$ of the deviation from their respective central tendencies have been undone. As such, we can safely regard the central tendencies of the output gap and inflation as long-run forecasts, where long run now can be interpreted as a forecast horizon of between five and ten years. ${ }^{8}$ The real interest rate has a much higher

\footnotetext{
${ }^{8}$ Note that the inclusion of the latent factors thus allows us to incorporate some measure of long-run output gap and inflation forecasts into the analysis. As argued by Kozicki and Tinsley (2001), includinig time-varying long-run forecasts (endpoints) is crucial to fitting the longer end of the yield curve. Interestingly, although we used a markedly different methodology, we obtain remarkably similar long-run inflation forecasts to the ones of Kozicki and Tinsley (2001), compare our Figure 3 with their Figure 6, panel B, p. 643. Also, we find that the Hoey survey long-run inflation expectations (collected from market participants) are remarkably close to our estimated long-run inflation expectation which corroborates even more our interpretation of central tendencies
} 
convergence speed. Around $95 \%$ of the deviation from its central tendency is undone after about nine months and more than $99 \%$ after one year and a half.

In order to corroborate the interpretation of the latent factors as long-run forecasts, we compare our results with the inflation forecasts from the Survey of Professional Forecasters provided by the Federal Reserve Bank of Philadelphia. Figure 5 shows the one-year ahead inflation forecast from this survey compared with the ones computed from our model. Figure 6 presents the comparison of the forecasts for the average rate of inflation for the next 10 years. Our estimates seem to track significantly well the patterns presented in the survey forecasts. The correlation between our model forecast and the survey forecast is equal to 0.94 and 0.92 for the one-year and ten-year forecast, respectively. More details regarding the computation of the inflation forecasts based on our model and the results from an ordinary least squares (OLS) regression of the survey forecast on our forecasts can be found in Appendix C.

Insert Figures 5 and 6

\subsubsection{Interpretation of standard "level" and "slope" effects}

Next, we turn to the interpretation of the factors in terms of their relation to the factors filtered from a completely latent setting. We follow Ang and Piazzesi (2000) in trying to interpret the factors of a fully latent three-factor Vasicek model (VM) in terms of the factors obtained in our model. The three factors in the VM can be labeled as one level effect and two slope effects. We did not find a curvature factor in this setting. We project the three Vasicek factors on our five factors. Regression results can be found in Table 4. Each of the VM latent factors can be reasonably explained in terms of the factors of our model, as indicated by the high $R^{2}$ statistics.

For the two VM slope factors, there is no clear interpretation in terms of a limited number of our factors. All five factors enter significantly in the slope regressions indicating that the VM slope factors are in fact a linear combination of our factors. Surprisingly, we do find a relatively straightforward interpretation of the VM level factor. In Figure 7, we present the contribution of the central tendency of inflation $\left(\pi^{*}\right)$ and the VM level factor subtracting the estimated constant to this factor. As can be seen, the correspondence between the VM level factor and our central tendency of inflation is extremely high. Although most of the other factors are statistically significant they add marginally to the explanation of the level factor. So, in contradiction to other papers, we do explain the level effect in terms of an affine transformation of a sensible macroeconomic variable: the central tendency of inflation.

Insert Figure 7

as long-run market forecasts. 
Table 4: Interpretation of latent Vasicek factors: OLS-based decomposition into factors

\begin{tabular}{|c|rrr|}
\hline \hline & Level factor & Slope factor 1 & Slope factor 2 \\
\hline cte & $-0.181(0.001)$ & $0.002(0.001)$ & $0.006(0.002)$ \\
$y$ & $-0.094(0.024)$ & $0.255(0.046)$ & $0.117(0.054)$ \\
$\pi$ & $0.267(0.036)$ & $0.826(0.068)$ & $-0.523(0.081)$ \\
$\rho$ & $0.217(0.036)$ & $0.560(0.069)$ & $-0.281(0.081)$ \\
$y^{*}$ & $2.989(0.306)$ & $-3.178(0.580)$ & $-10.810(0.686)$ \\
$\pi^{*}$ & $1.193(0.043)$ & $-0.931(0.082)$ & $0.470(0.097)$ \\
\hline $\bar{R}^{2}$ & 0.985 & 0.754 & 0.621 \\
\hline \hline
\end{tabular}

The three latent factors are regressed upon the filtered factors of the model presented above. Standard errors between brackets.

\subsubsection{The real interest rate rule}

Another observation to be made from Table 2 is that the standard Taylor rule in terms of observable variables does not hold for the real interest rate process. In fact, all the coefficients in the long-run real interest rate rule are not statistically significant. In order to gain some intuition into the implicit short-term rule for the real interest rate we re-arrange equation (3) as follows:

$$
\begin{aligned}
& E_{t} \mathrm{~d} \rho(t)=\kappa_{\rho \rho}\left(\rho_{s}^{*}-\rho(t)\right) \mathrm{d} t \\
& \rho_{s}^{*}=\underbrace{\gamma_{0}+\left(\gamma_{y}-\frac{\kappa_{\rho y}}{\kappa_{\rho \rho}}\right) y(t)+\left(\gamma_{\pi}-\frac{\kappa_{\rho \pi}}{\kappa_{\rho \rho}}\right) \pi(t)+\left(\gamma_{y^{*}}+\frac{\kappa_{\rho y}}{\kappa_{\rho \rho}}\right) y^{*}(t)+\left(\gamma_{\pi^{*}}+\frac{\kappa_{\rho \pi}}{\kappa_{\rho \rho}}\right) \pi^{*}(t)}_{\text {central bank short-term target }} .
\end{aligned}
$$

Within this framework, we can interpret $\rho_{s}^{*}$ as the time-variable short-term target level for the real interest rate of the central banker. Based on the estimates reported in Table 2, we have that rule is equal to (see also Figure 3):

$$
\rho_{s}^{*}=0.00005-0.037 y(t)-0.869 \pi(t)-0.171 y^{*}(t)+0.979 \pi^{*}(t)
$$

The above rule shows the importance of both the observed level of inflation and of the deviation from its central tendency. This rule can also be interpreted in terms of the responsiveness to deviations of actual output gap and inflation from its central tendencies and to the central tendencies themselves. As such, the implied target becomes:

$$
\rho_{s}^{*}=0.00005+0.037\left(y^{*}(t)-y(t)\right)+0.869\left(\pi^{*}(t)-\pi(t)\right)-0.209 y^{*}(t)+0.109 \pi^{*}(t)
$$

The real interest rate target increases as the central tendency of inflation goes up. Somewhat less conventional is the finding that the inflation deviation $\left(\pi^{*}-\pi\right)$ also enters with a positive sign. As such, higher (observed) inflation will ceteris paribus decrease the level of the real 
interest rate. Although at first sight this sign seems to be difficult to reconcile with intuition, it may be argued that it represents a term signaling the expected change in inflation. If the central tendency exceeds the current inflation rate, ceteris paribus, inflation will be expected to increase triggering a strong upward increase of the real interest rate, while if observed inflation exceeds its central tendency, ceteris paribus, inflation is expected to decrease (eventually) leading the central banker to loosen the contractionary policy stance. The real interest rate target seems much less responsive to the output variables. In this case, we find that a positive deviation, a shortfall of actual output to its central tendency, would tend to be associated with an increase in the real interest rate target. This result could be explained as a procyclical response of the central bank to expected increases in economic activity. We should, however, point out that both $\gamma_{y}$ and $\kappa_{\rho y}$ are not statistically significant.

Finally, there is the third feature of the real interest rate rule, which is related to the interest rate smoothing, i.e. to the mean reversion properties of the real interest rate to its target. We find, surprisingly, a very strong mean reversion for the real interest rate, i.e. a high value for $\kappa_{\rho \rho}$. The halving time of the deviation is less than three months. ${ }^{9}$ The extremely low halving time suggests that this policy reaction factor exerts more influence on the short end of the term structure than on the long end. In other words, the policy reaction factor represents a slope factor and not a level factor for the term structure. This is in line with the conjecture of Knez et al. (1994), Evans and Marshall (1998) and Wu (2000) that this policy reaction factor is intimately related to the slope of the yield curve.

\subsubsection{Term structure}

Finally, we can analyze the performance of the model in fitting the term structure of interest rates (Figure 8). The yield curve is affine in the state space vector. The loadings for the various maturities with respect to the factors are depicted in Figures 9 to 11. In contrast with the multi-factor latent factors literature, we do not find evidence in favor of the standard level effect. Instead we find a clear division between observable and non-observable (latent) factors. While observable factors almost exclusively exert their effects on the short end of the yield curve (Figure 10), latent factors affect the whole term structure significantly (except, by construction, for the very short run, see Figure 11). According to the factor loading estimates, yields with maturities over six months seem to be mainly responsive to changes in the central tendencies of inflation and output (note that observable inflation and output themselves only play a marginal role here). Since these central tendencies correspond to the long-run expectations (by construction), we can conclude from this that the longer end of the yield curve is mainly sensitive to long-run inflation and output forecasts. Inflation and

\footnotetext{
${ }^{9} \mathrm{~A}$ visual representation of the convergence speed is found in the next section where the impulse response functions are depicted and discussed.
} 
output forecasts, however, have different effects on the yield curve. Inflation forecast have almost identical effects across the yield curve, i.e. they constitute more of a level factor, while output forecasts exhibit a strong hump-shaped effect, affecting most strongly the intermediate maturities (from 6 months to about 2 to 3 years). For short-term maturities, and by definition, observable inflation and the real interest rate latent factor take over. The sensitivities of these variables decay quite fast with the time to maturity of the bond such that these factors can clearly be linked to the slope (spread) of the yield curve. While the estimated parameters turn out to be different over different subsamples (i.e. with a break point during the Volker period), the qualitative features of the factors remain the same, as can be inferred from the figures presented in Appendix B).

Insert Figures 9, 10 and 11

While observable macroeconomic variables do not constitute the major source in the dynamics of the yield curve itself, as can be inferred from the factor loadings, they still count as an important source of variation in the risk premia. As can be checked from Figure 12, risk premia for all maturities are volatile. Almost all time-varying risk premia parameters $(\Xi)$ are individually statistically significant. As such, time variability of risk premia adds statistically in modeling the term structure. Decomposing the risk premia into its different components, i.e. the risk premia related to the output gap, inflation, etc., shows that only the central tendency of output gap plays a minor role as a source of variation in the risk premia. Note that while each single component is in itself large, they often counterbalance each other, resulting in quite reasonable global (aggregate) risk premia.

\section{Insert Figure 12}

\subsection{Variance decomposition and impulse response analysis}

By construction, the Wiener shocks $d W_{i}(t), i=1, \ldots, n$ represent structural shocks related to each one of the variables (factors) in the model. Therefore, we can perform a meaningful variance decomposition and impulse response analysis on these shocks. The variance decomposition serves the purpose of finding out what type of shock is most important in moving the yield curve, while the impulse response analysis gives a detailed account of the cross-sectional and time series implications of each type of shock.

A general variance decomposition of yield curve changes over a horizon $h$ can be performed by decomposing the variance-covariance matrix into the responses to each of the different shocks. Defining a yield curve shock by $\Delta^{h} \hat{\mathbf{y}}(t) \equiv \hat{\mathbf{y}}(t+h)-\hat{\mathbf{y}}(t)$, it is easily shown that the variance-covariance matrix of these shocks takes the form: 


$$
\begin{aligned}
E_{t}\left(\left(\Delta^{h} \hat{\mathbf{y}}(t)-E_{t}\left(\Delta^{h} \hat{\mathbf{y}}(t)\right)\right)\left(\Delta^{h} \hat{\mathbf{y}}(t)-E_{t}\left(\Delta^{h} \hat{\mathbf{y}}(t)\right)\right)^{\prime}\right)= \\
\mathbf{B}\left[\int_{0}^{h} \exp (\mathbf{K}(s-h)) E_{t}\left(\mathbf{S S}^{\prime}\right) \exp (\mathbf{K}(s-h))^{\prime} \mathrm{d} s\right] \mathbf{B}^{\prime}
\end{aligned}
$$

The volatility over a given discrete time window of length $h$ of a specific yield curve maturity can be decomposed into the contributions of each of the orthogonal shocks. More specifically, the contribution of shocks of type $j$ in the variance of maturity $i$ over a time interval $h$ is given by:

$$
\sigma_{j}^{2} \int_{0}^{h}\left(\sum_{k=1}^{n} \mathbf{B}_{k}(i)(\exp (\mathbf{K}(s-h)))_{k, j}\right)^{2} \mathrm{~d} s .
$$

Table 5 presents the steady state variance decomposition for different horizons of the yield curve in terms of the five possible structural shocks. The estimates suggest that the variability across the yield curve is basically explained by three types of shocks: shocks to the real interest rate and to the central tendencies of output gap and inflation. Actual output gap and observed inflation series explain at most $6 \%$ of the variability of yield curve movements. Also, we find a strong distinction between the types of shocks affecting the short end and the longer end of the yield curve. The shorter end (say maturities up to two years) is dominated by both the central tendency of output and real interest rate shocks, with a strong emphasis on the former type of shock. The variability in the longer end of the yield curve, in contrast, is mainly determined by the long-run inflation tendency.

Impulse response analysis provides a complementary tool to gauge the importance of the various types of shocks for the macroeconomy and the yield curve. This tool analyses the predictable responses to the various shocks. We follow the standard literature by analyzing the effects of a one standard deviation shock in each of these sources of uncertainty. All figures are presented as deviations from the base line case.

Figures 13 to 17 present the standard impulse response functions (IRFs) for output gap, inflation, the real interest rate and the central tendencies of output gap and inflation. A positive output gap shock (Figure 13) generates a cyclical response to most of the variables involved with and initial increase in inflation. The yield curve response to this type of shock is represented by an initial upward tilting of the short end of the curve (see also Figure 18). A temporary inflation shock (Figure 14) generates a negative but marginal output effect. Again, because of the temporary nature of the shock, the yield curve response is limited to an upward tilting of the shorter end of the curve. A positive real interest rate shock (Figure 15) generates marginal effects on both the output gap and on inflation. A shock in the 
Table 5: Yield curve variance decomposition at different horizons in the steady state

\begin{tabular}{|c|c|c|c|c|c|c|}
\hline in $\%$ & $\mathbf{y}$ & $\pi$ & $\rho$ & $\mathrm{y}^{*}$ & 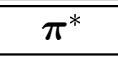 & total \\
\hline \multicolumn{7}{|c|}{ time horizon: $h=d t$} \\
\hline yield $_{\mathbf{1 q}}$ & 5.270 & 1.764 & 28.413 & 52.454 & 12.100 & 100.00 \\
\hline $\operatorname{yield}_{\mathbf{2 q}}$ & 6.265 & 0.702 & 8.081 & 67.300 & 17.652 & 100.00 \\
\hline yield $_{1 \mathbf{y r}}$ & 5.383 & 0.213 & 2.308 & 69.013 & 23.082 & 100.00 \\
\hline yield $\mathbf{2 y r}_{\mathbf{y r}}$ & 3.274 & 0.018 & 0.762 & 63.514 & 32.432 & 100.00 \\
\hline $\operatorname{yield}_{5 \mathbf{y r}}$ & 0.663 & 0.016 & 0.211 & 42.511 & 56.599 & 100.00 \\
\hline yield 10yr & 0.189 & 0.007 & 0.077 & 19.898 & 79.828 & 100.00 \\
\hline \multicolumn{7}{|c|}{ time horizon: $h=1$ year } \\
\hline yield $_{1 q}$ & 5.872 & 1.209 & 18.024 & 57.719 & 17.177 & 100.00 \\
\hline $\operatorname{yield}_{\mathbf{2 q}}$ & 6.031 & 0.438 & 4.830 & 67.431 & 21.270 & 100.00 \\
\hline yield $1 \mathbf{y r}$ & 4.876 & 0.117 & 1.426 & 67.392 & 26.189 & 100.00 \\
\hline $\operatorname{yield}_{2 \mathbf{y r}}$ & 2.805 & 0.005 & 0.505 & 61.162 & 35.524 & 100.00 \\
\hline yield $_{5 \mathbf{y r}}$ & 0.506 & 0.027 & 0.146 & 39.903 & 59.418 & 100.00 \\
\hline yield $10 y r$ & 0.139 & 0.011 & 0.051 & 18.252 & 81.547 & 100.00 \\
\hline
\end{tabular}

central tendency of output (Figure 16) triggers a positive output gap and a negative inflation response accompanied by a significant downward tilting of the yield curve. Finally, a shock to the long-run tendency of inflation (Figure 17) generates a positive output and inflation responses and generates a level shift of the entire yield curve, in contrast to the other shocks. In this case, the high effect of the shock is due to its high degree of inertia.

\section{Insert Figures 13 to 17}

We focus now on the effects of the different shocks on the evolution of the term structure of interest rates (Figure 18). It is easily seen that the term structure variability is primarily determined by the central tendencies of output gap and inflation shocks. However, both the dynamic effects as well as the effect across maturities are different between these shocks. For the shocks to the central tendency of output, i.e. shocks to the output capacity, we find a gradual decrease of the impact of the shock through time. Simulation suggests that the maximal effect is reached at about two quarters, whereafter the term structure starts converging back towards the benchmark. Note that because of the relatively strong mean reversion of interest rates, the effects of this type of shock will be most pronounced at the shorter end of the term structure. As such, this type of shock essentially represents a yield curve tilting shock.

Shocks to the central tendency of inflation differ drastically in their effect from output gap shocks. Due to the extremely low mean reversion in the central tendency of inflation, such a shock will be effective over a much longer time period. Hence, this type of shock affects the 
entire term structure and causes a shift over the entire maturity spectrum. Moreover, this type of shock has long lasting effects on the term structure as can be observed from the last panel of Figure 18. Even five years after the shock, no noticeable convergence towards the benchmark can be detected.

Finally, we also find some effects of output gap and real interest rate shocks on the short end of the term structure. However, the size of these shocks is markedly different from the shocks to the central tendencies.

\section{Insert Figure 18}

\subsection{Forecast evaluation}

Next to the cross-sectional issues relating the macroeconomy to the term structure and viceversa we analyze the fit of the time series dimension of the model in more detail. We analyze specifically two issues: the forecasting performance of the structural model at various horizons and the quality of the forecasts, i.e. an analysis of the biases in these forecasts.

Starting with the forecasting performance, we compare the forecasting behavior of the model against three alternatives: the random walk model, an AR(1) model and a VAR(1) representation (in output gap, inflation and the three-month interest rate). First, we test the forecasting performance for both output and inflation. Taking the root mean square error (RMSE) as the measure we depict the forecasting performance in Figure 19.

This figure sets out the ratio between the RMSE of the model and each of the three alternatives against the prediction horizon. The prediction horizon is stated in quarters. We thus analyze forecasting performance for horizons from one quarter up to five years. The first panel of the figure shows the predictive performance for the output gap and the second panel for inflation. The results indicate that the model has a certain predictive performance. That is, the alternative of no predictive power, the random walk model, is clearly outperformed by our model with reductions in the RMSE up to almost $50 \%$ for output and about 10 to $15 \%$ for inflation. Also, as long as the forecasting horizons stay within a reasonable range, the model performs better than standard AR(1) representations for both the output gap and inflation. The third alternative (VAR), however, shows that the model is not superior on both accounts. The model does consistently worse than the VAR representation for inflation, while it does marginally better in predicting output for the forecasting horizon above 1 year.

\section{Insert Figure 19}

We also analyze the forecast performance for future yield curve evolutions. Here we take as a relevant benchmark the random walk model as it has been shown in the literature that this alternative typically outperforms the predictions based on (latent) multi-factor models. Figure 19 (lower-left panel) sets out the RMSE ratio of the model relative to the random 
walk predictions. As can be seen from this figure, we find that for short-term predictions we face the same difficulties in beating the random walk model as the standard multi-factor models. Note, however, that alternative representations including macro variables (as in Ang and Piazzesi, 2000) also report this prediction failure at short horizons. Nevertheless, at longer prediction horizons, depending as well on the maturity being predicted, the model starts to outperform the random walk model. Typically, the superior performance starts for prediction horizons exceeding one to two years. Finally, if we take the forecast performance of the latent three factor Vasicek model as a benchmark one can see that our model outperforms this benchmark especially between the prediction horizon of 2 quaters to 3 years (Figure 20).

\section{Insert Figure 20}

A second issue concerns the quality of the forecasts. We test for the unbiasedness of the forecasts through regressions of the actual values (data) on a constant and forecasted values. Unbiasedness of the forecasts would imply a zero constant and a regression coefficient on the forecasted values equal to one. Table 6 presents the results of this regression for the output gap, inflation and yield curve predictors. As can be inferred from this table, we cannot reject the hypothesis that the model generates unbiased predictors for output and inflation in the short-run (up to 2 quarters for output and 4 quarters for inflation). However, extending the prediction horizon beyond 2 quarters, the quality of the predictors deteriorates. For all series considered, we detect significant biases in the predictions. Note, however, that this bias is not only present in the model's predictions but also in each of the alternatives to the model considered. Another remark to be made in this respect is that the forecasts, although biased, still detect some significant pattern. All of the listed coefficients are significantly different from zero, indicating at least a positive covariation between the actual and the forecasted series. The bottom line thus seems to be that although the model yields statistically relevant forecasts, in terms of RMSE reductions, the model does not pass the test of generating optimal, in the sense of unbiased, predictors. Finding better predictors, generated by better models, remains one of the possible routes for future research. 
Table 6: Regressions to test for unbiasedness of forecasts

\begin{tabular}{|c|c|c|c|c|c|c|}
\hline series & Model & $2 q$ & $4 q$ & $6 q$ & $8 q$ & $12 q$ \\
\hline \multirow{3}{*}{$\mathbf{y}$} & $\overline{\text { Macro }}$ & $\bar{~} 1.13$ & $0.70^{*}$ & $0.53^{* *}$ & 0.71 & $\overline{0.92}$ \\
\hline & $\mathbf{A R}(\mathbf{1})$ & 0.90 & $0.45^{* *}$ & $-0.24^{* *}$ & $-0.94^{* *}$ & $-2.26^{* *}$ \\
\hline & $\operatorname{VAR}(\mathbf{1})$ & 0.92 & $0.61^{* *}$ & $0.33^{* *}$ & $0.38^{* *}$ & $0.61^{*}$ \\
\hline \multirow{3}{*}{$\pi$} & Macro & 0.98 & 0.92 & $0.81^{* *}$ & $0.61^{* *}$ & $0.19^{* *}$ \\
\hline & $\mathbf{A R}(\mathbf{1})$ & 0.97 & $0.87^{* *}$ & $0.73^{* *}$ & $0.58^{* *}$ & $0.42^{* *}$ \\
\hline & VAR(1) & 0.99 & 0.95 & $0.88^{*}$ & $0.74^{* *}$ & $0.34^{* *}$ \\
\hline \multirow[t]{2}{*}{ yield $_{1 q}$} & Macro & $0.91^{* *}$ & $0.80^{* *}$ & $0.66^{* *}$ & $0.55^{* *}$ & $0.39^{* *}$ \\
\hline & R.W. & $0.87 * *$ & $0.79^{* *}$ & $0.69^{* *}$ & $0.56^{* *}$ & $0.38^{* *}$ \\
\hline \multirow[t]{2}{*}{ yield $_{2 q}$} & Macro & $0.90^{* *}$ & $0.79^{* *}$ & $0.65^{* *}$ & $0.54^{* *}$ & $0.39 * *$ \\
\hline & R.W. & $0.87 * *$ & $0.80^{* *}$ & $0.69^{* *}$ & $0.57^{* *}$ & $0.40^{* *}$ \\
\hline \multirow[t]{2}{*}{ yield $_{1 \mathrm{yr}}$} & Macro & $0.90^{* *}$ & $0.80^{* *}$ & $0.67^{* *}$ & $0.57^{* *}$ & $0.42^{* *}$ \\
\hline & R.W. & $0.89 * *$ & $0.81^{* *}$ & $0.71^{* *}$ & $0.61^{* *}$ & $0.46^{* *}$ \\
\hline \multirow[t]{2}{*}{ yield $_{2 y r}$} & Macro & $0.92^{* *}$ & $0.82^{* *}$ & $0.71^{* *}$ & $0.63^{* *}$ & $0.49 * *$ \\
\hline & R.W. & $0.90^{* *}$ & $0.83^{* *}$ & $0.74^{* *}$ & $0.67^{* *}$ & $0.55^{* *}$ \\
\hline \multirow[t]{2}{*}{ yield $_{5 y r}$} & Macro & $0.93^{* *}$ & $0.85^{* *}$ & $0.76^{* *}$ & $0.70^{* *}$ & $0.58^{* *}$ \\
\hline & R.W. & $0.93^{* *}$ & $0.86^{* *}$ & $0.79^{* *}$ & $0.74^{* *}$ & $0.66^{* *}$ \\
\hline \multirow[t]{2}{*}{ yield $_{10 y r}$} & Macro & $0.94^{* *}$ & $0.86^{* *}$ & $0.79^{* *}$ & $0.73^{* *}$ & $0.63^{* *}$ \\
\hline & R.W. & $0.94^{* *}$ & $0.89^{* *}$ & $0.83^{* *}$ & $0.80^{* *}$ & $0.73^{* *}$ \\
\hline
\end{tabular}

Macro in the above table refers to the macroeconomic model as set out in Section 2. ${ }^{*}$ Significantly different from 1 at $10 \%$ level. ${ }^{* *}$ Significantly different from 1 at $5 \%$ level. 


\section{Conclusions}

In this paper we have presented a methodology to estimate a continuous time model of the term structure incorporating both observable as well as latent factors in the state vector. The framework is flexible enough so that the state space can be increased to include other observable or non-observable factors and to encompass a wide variety of interest rate rules in both these factors. As such, it is well suited to tackle questions related to the interrelations between financial markets and the macroeconomy.

We used the methodology to estimate a feedback rule on the entire term structure of interest rates. The model estimated in this paper is akin to the standard view in the monetary literature on Taylor rules. More specifically, we used the standard aggregate demand-aggregate supply literature to identify the macroeconomic dynamics and imposed the feedback rule as a long-run attractor for the real interest rate. Estimating the model, we find that the standard feedback rule in observed inflation and output is not retained as a valid description of the bond markets. Observed inflation and output typically do not have the dynamic properties required to fit cross-sectionally the term structure of interest rates. Instead, we find evidence in favor of a different type of short-term real interest rate rule where the central banker reacts both to a (latent) central tendency of inflation and to the gap between observed inflation and this central tendency. In particular, movements of interest rates are basically one-to-one with the central tendency of inflation, which in its turn can be interpreted as a long-run inflation forecast.

Obviously, a number of caveats needs to be taken into account. First, our model represents an economy with perfect financial markets. That is, we have assumed from the start the absence of market segmentation. Moreover, we have assumed that the prices of risk are captured within the framework of essentially affine models. Both these assumptions may be a serious oversimplification of reality and may affect the estimated Taylor-type rule. They obviously also set out a research agenda. For instance, an interesting extension of this model is to consider a broader class of models (for instance, the class of quadratic term structure models, QTSMs, see Ahn et al. (2000)), or to consider more complex dynamic structures for the macroeconomy. Alternatively, we could start to explicitly estimate general equilibrium models incorporating both the macroeconomy and the bond markets within the framework of affine continuous time models, along the lines of Berardi (2001) and Wu (2000). These possible extensions are left for future research. 


\section{References}

[1] Ahn, D., R. Dittmar and R. Gallant (2000), "Quadratic Term Structure Models: Theory and Evidence", forthcoming in Review of Financial Studies, spring 2002.

[2] Ang A. and M. Piazzesi (2001), "A No-Arbitrage Vector Autoregression of Term Structure Dynamics with Macroeconomic and Latent Variables", NBER working paper 8363.

[3] Bakshi, G. S. and Z. Chen, (1996), "Inflation, Asset Prices and the Term Structure of Interest Rates in Monetary Economies", Review of Financial Studies 9, no. 1, 241-275.

[4] Beaglehole, D. R. and M. S. Tenney (1991), "General Solutions of Some Interest RateContingent Claim Pricing Equations", The Journal of Fixed Income, 69-83.

[5] Berardi, A. (2001), "How Strong is the Relation Between the Term Structure, Inflation and GDP?", working paper.

[6] Bliss, R. (1997) "Testing Term Structure Estimation Methods", Advances in Futures and Options Research 9, 197-231.

[7] Bollerslev, T. and J. Wooldridge (1992), "Quasi-Maximum Likelihood Estimation and Inference in Dynamic Models with Time-Varying Covariances", Econometric Reviews 11, 143-172.

[8] Buraschi, A. (1996), "How Large is the Inflation Risk Premium in the U.S. Nominal Term Structure?", working paper, IFA London Business School, no. 232.

[9] Chen, R.-R. and L. Scott (1993), "Maximum Likelihood Estimation for a Multi-Factor Equilibrium Model of the Term Structure of Interest Rates", Journal of Fixed Income, December, 14-31.

[10] Clarida, R., J.Galí and M. Gertler (1998), "Monetary Policy Rules in Practice. Some International Evidence", European Economic Review 42, 1033-1067.

[11] Dai, Q. and K. Singleton (2000), "Specification Analysis of Affine Term Structure Models", Journal of Finance, v. LV, 1943-1978, also appeared as NBER working paper 6128 (1997).

[12] Dai, Q. and K. Singleton (2001), "Expectation Puzzles, Time-Varying Risk Premia, and Affine Models of the Term Structure", Journal of Financial Economics, forthcoming.

[13] de Jong, F. (2000), "Time Series and Cross-section Information in Affine Term-Structure Models", Journal of Business and Economic Statistics, 18, no. 3, 300-314. 
[14] Duan, J.-C. and J.-G. Simonato (1999), "Estimating and Testing Exponential-Affine Term Structure Models by Kalman Filter", Review of Quantitative Finance and Accounting, 13, 2, 111-135.

[15] Duffee, G. (2001), "Term Premia and Interest Rate Forecasts in Affine Models", working paper, Haas School of Business, forthcoming in Journal of Finance.

[16] Duffee, G. and R. Stanton (2001), "Estimation of Dynamic Term Structure Models", working paper, Haas School of Business, U.C. Berkeley.

[17] Fleming, M. and E. Remolona (2001), "The Term Structure of Announcement Effects", working paper.

[18] Gallant, A. and G. Tauchen (1992), "A Nonparametric Approach to Nonlinear Time Series Analysis: Estimation and Simulation", in D. Brillinger, P. Cains, J. Geweke, E. Parzen, M. Rosenblatt, and M. Taqqu, eds., New Directions in Time Series Analysis, Part II, New York: Springer Verlag, 71-92.

[19] Harvey, A. C. (1989), Forecasting, Structural Time Series Models and Kalman Filter, Cambridge University Press.

[20] Knez P., Litterman R. and J. A. Scheinkman (1994), "Explorations into Factors Explaining Money Market Returns", Journal of Finance, 49, 1861-1882.

[21] Kozicki S. and P.A. Tinsley (2001) Shifting endpoints in the terms structure of interest rates, Jounrnal of Monetary Economics, 47, 613-652.

[22] McCulloch, J. and H. Kwon (1993), "U.S. Term Structure Data, 1947-1991", Ohio State University Working Paper 93-6.

[23] Moler, C. and C. Van Loan (1978), "Nineteen Dubious Ways to Compute the Exponential of A Matrix", SIAM Review 20, no. 4, 801-836.

[24] Pearson, N. and T. Sun (1994), "Exploiting the Conditional Density in Estimating the Term Structure : An Application to the Cox, Ingersoll and Ross Model", Journal of Finance 49, no. 4, 1279 - 1304.

[25] Piazzesi, M. (2001), "An Econometric Model of the Yield Curve with Macroeconomic Jump Effects", NBER working paper 8246.

[26] Wu, T., (2000), "Macro Factors and the Affine Term Structure of Interest Rates", working paper. 


\section{Appendix A: Computing the exponential of a matrix}

In this appendix, we present the procedure used in this paper to generate the conditional expectations and variance covariance matrix for the levels of the factors. In essence, the proposed procedure solves the problem of the computation of an exponential of a matrix (see Moler and Van Loan (1978)). The procedure we follow is to compute the variance-covariance matrix from the raw first and second moments:

$$
\operatorname{cov}_{t}\left(f_{i}(s), f_{j}(s)\right)=E\left(f_{i}(s) f_{j}(s) \mid \mathbf{f}(t)\right)-E\left(f_{i}(s) \mid \mathbf{f}(t)\right) E\left(f_{j}(s) \mid \mathbf{f}(t)\right)
$$

with $s=t+h$. We, therefore, need to compute the conditional expectations for the factors and all of its cross-products for the time horizon $h$ (equal to one quarter in our case) over any desired time interval $t$. For this purpose, we first construct the $q \times 1$ vector $\mathbf{g}$ containing the expectations of the factors, its squares and all possible cross-products:

$$
\begin{aligned}
\mathbf{g}(s) \equiv E_{t}^{P}\left(f_{1}(s), \ldots, f_{5}(s), f_{1}^{2}(s), \ldots, f_{5}^{2}(s), f_{1}(s) f_{2}(s), \ldots, f_{1}(s) f_{5}(s)\right. \\
\\
\left.f_{2}(s) f_{3}(s), \ldots, f_{2}(s) f_{5}(s), f_{3}(s) f_{4}(s), f_{3}(s) f_{5}(s), f_{4}(s) f_{5}(s) \mid \mathbf{f}(t)\right)^{\prime}
\end{aligned}
$$

The dynamics for this state vector can be constructed by means of Ito's lemma. First, for the first four rows, i.e. the factors themselves, we have the assumed dynamics

$$
\mathrm{d} \mathbf{f}(s)=\mathbf{K}(\boldsymbol{\psi}-\mathbf{f}(s)) \mathrm{d} s+\mathbf{S} \mathrm{d} \mathbf{W}(s) .
$$

which, taking expectations, gives:

$$
\begin{aligned}
& E_{t}^{P}\left[d f_{i}(s)\right]=E_{t}^{P}\left[\sum_{j=1}^{n} K_{i, j}\left(\Psi_{j, 1}-f_{j, 1}(s)\right) \mathrm{ds}+S_{i, j} d W_{j, 1}(s)\right] \\
& E_{t}^{P}\left[d f_{i}(s)\right]=\sum_{j=1}^{n} K_{i, j}\left(\Psi_{j, 1}-E_{t}^{P}\left(f_{j, 1}(s)\right)\right) \mathrm{ds}
\end{aligned}
$$

for $i=1, \ldots, n$. Using the fact that $E_{t}^{P}\left[d f_{i}(s)\right]=d\left(E_{t}^{P}\left[f_{i}(s)\right]\right)$ and the definitions in Eq.(28) we obtain the first $n$ elements of the vector $\mathrm{dg}$ :

$$
d g_{i}(s)=\sum_{j=1}^{n} K_{i, j}\left(\Psi_{j, 1}-g_{j}(s)\right) \mathrm{ds}
$$

For the following $n$ elements of the vector $\mathbf{g}$ which contain the square of the factors, we have (applying Ito's Lemma): 


$$
\begin{aligned}
& E_{t}^{P}\left[d f_{i}^{2}(s)\right]=E_{t}^{P}\left[2 f_{i}(s) d f_{i}(s)+d f_{i}^{2}(s)\right] \\
& E_{t}^{P}\left[d f_{i}^{2}(s)\right]=E_{t}^{P}\left[2 f_{i}(s)\left(\sum_{j=1}^{n} K_{i, j}\left(\Psi_{j, 1}-f_{j, 1}(s)\right) \mathrm{ds}+S_{i, j} d W_{j, 1}(s)\right)+\left(\sum_{j=1}^{n} S_{i, j} d W_{j, 1}(s)\right)^{2}\right]
\end{aligned}
$$

for $i=1, \ldots, n$. Since $S$ is a diagonal matrix and applying the same reasoning as before we get the following:

$$
d g_{n+i}(s)=\left\{2 g_{i}(s) \sum_{j=1}^{n} K_{i, j} \Psi_{j, 1}-2\left(\sum_{j=1}^{n} K_{i, j} E_{t}^{P}\left[f_{i}(s) f_{j}(s)\right]\right)+E_{t}^{P} S_{i, i}^{2}\right\} d s
$$

for $i=1, \ldots, n$. We construct the following auxiliary matrix to help identify the appropriate elements of the vector $\mathrm{g}$ :

$$
m=\left(\begin{array}{lllll}
n+1 & n+6 & n+7 & n+8 & n+9 \\
n+6 & n+2 & n+10 & n+11 & n+12 \\
n+7 & n+8 & n+3 & n+13 & n+14 \\
n+9 & n+10 & n+11 & n+4 & n+15 \\
n+12 & n+13 & n+14 & n+15 & n+5
\end{array}\right)
$$

and obtain:

$$
d g_{m_{i, i}}(s)=d g_{n+i}(s)=\left\{2 g_{i}(s) \sum_{j=1}^{n} K_{i, j} \Psi_{j, 1}-2\left(\sum_{j=1}^{n} K_{i, j} g_{m_{i, j}}\right)+S_{i, i}^{2}\right\} d s
$$

for $i=1, \ldots, n$. For the final elements of $\mathbf{g}$ containing the cross-terms of the factors, we have that:

$$
E_{t}^{P}\left[d\left(f_{i}(s) f_{j}(s)\right)\right]=E_{t}^{P}\left[f_{j}(s) d f_{i}(s)+f_{i}(s) d f_{j}(s)+d f_{i}(s) d f_{j}(s)\right]
$$

Assuming that $E_{t}^{P}\left[d W_{i}(s) d W_{j}(s)\right]=0$ for $i, j=1, \ldots, n$ and $i \neq j$, we obtain:

$$
\begin{aligned}
E_{t}^{P}\left[d\left(f_{i}(s) f_{j}(s)\right)\right]= & E_{t}^{P}\left[f_{j}(s)\left(\sum_{k=1}^{n} K_{i, k}\left(\Psi_{k, 1}-f_{k, 1}(s)\right) \mathrm{d} s+S_{i, k} d W_{k, 1}(s)\right)\right. \\
& \left.+f_{i}(s)\left(\sum_{n=1}^{n} K_{j, n}\left(\Psi_{n, 1}-f_{n, 1}(s)\right) \mathrm{d} \mathrm{s}+S_{j, n} d W_{n, 1}(s)\right)\right]
\end{aligned}
$$

And making use again of the auxiliary matrix m gives:

$$
d g_{m_{i, j}}(s)=\left[g_{j}(s) \sum_{k=1}^{n} K_{i, k}\left(\Psi_{k, 1}-g_{m_{j, k}}(s)\right)+g_{i}(s) \sum_{n=1}^{n} K_{j, n}\left(\Psi_{n, 1}-g_{m_{i, n}}(s)\right)\right] d s
$$

Note that the dynamics can be restated in terms of the vector $\mathbf{g}$ :

$$
\mathrm{d} \mathbf{g}(s)=(\Theta+\boldsymbol{\Omega} \mathbf{g}(s)) \mathrm{ds} .
$$

Since the conditional expectations contained in the vector $\mathrm{g}$ are computed for each point in time $t$, we have that $d s=d h$ which gives the following solution: 


$$
\mathbf{g}(t+h)=-\boldsymbol{\Omega}^{-\mathbf{1}} \Theta+\mathbf{e}^{\boldsymbol{\Omega} h}\left(\mathrm{~g}(t)+\mathbf{\Omega}^{-\mathbf{1}} \Theta\right)
$$

Making $\boldsymbol{\Gamma}(h)=\mathbf{e}^{\boldsymbol{\Omega} h}$ gives

$$
\mathbf{g}(t+h)=-\boldsymbol{\Omega}^{-\mathbf{1}} \Theta+\boldsymbol{\Gamma}(h)\left(\mathbf{g}(t)+\boldsymbol{\Omega}^{-\mathbf{1}} \Theta\right)
$$

The above equation shows that in order to compute the conditional expectations and the variance covariance matrix of the factors, we basically need to compute the matrix $\boldsymbol{\Gamma}(h)$. 


\section{Appendix B: Estimation results for the subperiods 1958-1979 and 1982-1998}

Table B1: Maximum likelihood estimates (1958:Q1-1979:Q2)

\begin{tabular}{|c|c|c|c|c|c|c|}
\hline & $\mathrm{y}$ & $\pi$ & $\rho$ & $\mathbf{y}^{*}$ & $\pi^{*}$ & \\
\hline $\boldsymbol{\kappa}_{y,}$ & $\begin{array}{c}1.2902 \\
(0.2196)\end{array}$ & $\begin{array}{c}0.1926 \\
(0.4196)\end{array}$ & $\begin{array}{l}-0.2255 \\
(0.1863)\end{array}$ & & & \\
\hline $\boldsymbol{\kappa}_{\pi,}$ & $\begin{array}{l}-0.5941 \\
(0.1796)\end{array}$ & $\begin{array}{l}-1.3577 \\
(1.0978)\end{array}$ & $\begin{array}{l}-0.8329 \\
(0.2193)\end{array}$ & & & \\
\hline$\kappa_{\rho,}$ & $\begin{array}{l}-0.0074 \\
(0.0201)\end{array}$ & $\begin{array}{c}2.1638 \\
(0.9941)\end{array}$ & $\begin{array}{c}1.0920 \\
(0.3557)\end{array}$ & & & \\
\hline $\boldsymbol{\kappa}_{y^{*}}$ & & & & $\begin{array}{c}0.3643 \\
(0.0507)\end{array}$ & & \\
\hline $\boldsymbol{\kappa}_{\pi^{*}}$ & & & & & $\begin{array}{c}0.0012 \\
(0.0010) \\
\end{array}$ & \\
\hline$\theta$. & & & & $\begin{array}{l}-0.00005 \\
(0.00007)\end{array}$ & $\begin{array}{l}0.028524 \\
(0.02835)\end{array}$ & \\
\hline$\gamma_{0}$ & & & $\begin{array}{c}0.00006 \\
(0.00018)\end{array}$ & & & \\
\hline$\gamma$ & $\begin{array}{l}-0.2148 \\
(0.1896)\end{array}$ & $\begin{array}{c}0.9595 \\
(1.1066)\end{array}$ & & $\begin{array}{c}-0.0943 \\
(0.2010)\end{array}$ & $\begin{array}{l}-1.3507 \\
(1.0346)\end{array}$ & \\
\hline$\lambda$ & $\begin{array}{c}58.4377 \\
(19.8123)\end{array}$ & $\begin{array}{c}-3.4210 \\
(343.5178)\end{array}$ & $\begin{array}{c}-9.2260 \\
(1579.0654)\end{array}$ & & & \\
\hline$\Xi_{\rho,}$ & $\begin{array}{l}-5.4505 \\
(1.5992)\end{array}$ & $\begin{array}{l}14.9656 \\
(2.6784)\end{array}$ & $\begin{array}{l}17.6682 \\
(2.8740)\end{array}$ & $\begin{array}{c}485.0702 \\
(445.6150)\end{array}$ & $\begin{array}{l}-21.8112 \\
(4.4287)\end{array}$ & \\
\hline$\sigma^{2}$ & $\begin{array}{c}0.000467 \\
(5.918679)\end{array}$ & $\begin{array}{c}0.000048 \\
(5.451926)\end{array}$ & $\begin{array}{c}0.000086 \\
(0.786101)\end{array}$ & $\begin{array}{c}0.0000004 \\
(0.60301)\end{array}$ & $\begin{array}{c}0.000026 \\
(4.679106)\end{array}$ & \\
\hline $\mathbf{R}_{1 q}$ & 15.1138 & & & & & \\
\hline $\mathbf{R}_{2 q}$ & 10.6568 & 9.4902 & & & & \\
\hline $\mathbf{R}_{1 y r}$ & 3.7122 & 4.1557 & 2.7524 & & & \\
\hline $\mathbf{R}_{2 y r}$ & 0.0593 & 0.0002 & -0.0001 & 0.0000 & & \\
\hline $\mathbf{R}_{5 y r}$ & -0.01182 & -0.4215 & -0.0001 & 0.5717 & 1.9994 & \\
\hline $\mathbf{R}_{10 y r}$ & 0.0972 & -0.0727 & -0.0071 & 0.1158 & 0.6143 & 0.0007 \\
\hline
\end{tabular}

ML estimates with standard errors underneath. Only the lower diagonal of the measurement error covariance matrix is given. These values are multiplied by $10^{6}$. Total loglikelihood amounts to 3766.3171 or 43.7944 on average (excluding constant in the loglikelihood). 
Table B2: Maximum likelihood estimates (1982:Q1-1998:Q4)

\begin{tabular}{|c|c|c|c|c|c|c|}
\hline & $\mathbf{y}$ & $\pi$ & $\rho$ & $\mathbf{y}^{*}$ & $\pi^{*}$ & \\
\hline \multirow[t]{2}{*}{$\boldsymbol{\kappa}_{y,}$} & 0.4768 & 0.0338 & -0.5039 & & & \\
\hline & $(0.2577)$ & $(0.2448)$ & $(0.3507)$ & & & \\
\hline \multirow{2}{*}{$\boldsymbol{\kappa}_{\pi,}$} & -0.2561 & -0.3999 & -1.2006 & & & \\
\hline & $(0.2990)$ & $(0.5240)$ & $(0.2986)$ & & & \\
\hline \multirow{2}{*}{$\boldsymbol{\kappa}_{\rho,}$} & -0.0074 & 0.6071 & 1.3389 & & & \\
\hline & $(0.0436)$ & $(0.6594)$ & (1.3134) & & & \\
\hline \multirow[t]{2}{*}{$\boldsymbol{\kappa}_{y^{*},}$} & & & & 0.6956 & & \\
\hline & & & & $(0.1265)$ & & \\
\hline \multirow{2}{*}{$\boldsymbol{\kappa}_{\pi^{*},}$} & & & & & 0.0083 & \\
\hline & & & & & $(0.0022)$ & \\
\hline \multirow[t]{2}{*}{$\theta$. } & & & & -0.00006 & 0.07962 & \\
\hline & & & & $(0.00055)$ & $(0.01325)$ & \\
\hline \multirow[t]{2}{*}{$\gamma_{0}$} & & & 0.00006 & & & \\
\hline & & & $(0.00075)$ & & & \\
\hline \multirow[t]{2}{*}{$\gamma$} & 0.0370 & 0.5501 & & -0.0348 & 0.0954 & \\
\hline & $(0.2599)$ & $(0.4203)$ & & $(0.1950)$ & $(0.5362)$ & \\
\hline \multirow[t]{2}{*}{$\lambda$} & 46.8373 & -3.8778 & -9.2563 & & & \\
\hline & $(194.3650)$ & $(92.5728)$ & $(4498.3324)$ & & & \\
\hline \multirow{2}{*}{$\Xi_{\rho,}$} & -5.6896 & 17.6764 & 32.7754 & 498.0588 & -94.5289 & \\
\hline & $(7.3782)$ & $(5.2057)$ & $(7.5508)$ & (1446.1787) & $(30.4351)$ & \\
\hline \multirow[t]{2}{*}{$\sigma^{2}$} & 0.000112 & 0.000090 & 0.000223 & 0.000001 & 0.000010 & \\
\hline & (0.000019) & $(0.000022)$ & $(0.000280)$ & $(0.000004)$ & $(0.000004)$ & \\
\hline $\mathbf{R}_{1 q}$ & 16.3199 & & & & & \\
\hline $\mathbf{R}_{2 q}$ & 10.6688 & 8.6115 & & & & \\
\hline $\mathbf{R}_{1 y r}$ & 3.7621 & 4.1420 & 3.0511 & & & \\
\hline $\mathbf{R}_{2 y r}$ & 0.0593 & 0.0002 & -0.0001 & 0.0000 & & \\
\hline $\mathbf{R}_{5 y r}$ & -0.1167 & -0.4236 & -0.0001 & 0.5708 & 1.9034 & \\
\hline $\mathbf{R}_{10 y r}$ & 0.0972 & -0.0727 & -0.0071 & 0.1173 & 0.6155 & 0.0007 \\
\hline
\end{tabular}

ML estimates with standard errors underneath. Only the lower diagonal of the measurement error covariance matrix is given. These values are multiplied by $10^{6}$. Total loglikelihood amounts to 2828.8282 or 44.2004 on average (excluding constant in the loglikelihood). 


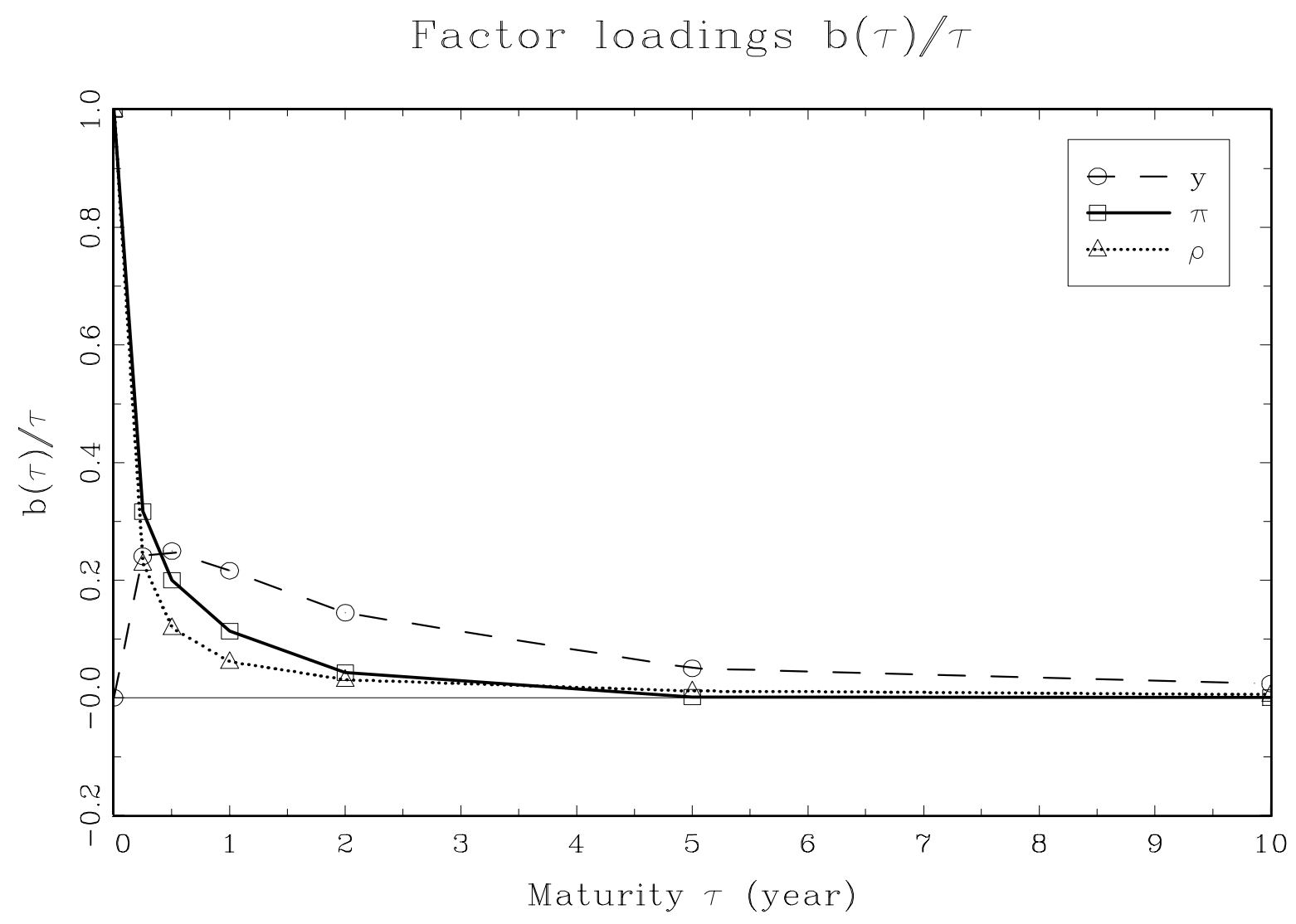

Figure B1: Estimated factor loadings- Factors 1 to 3 (1958-1979). 


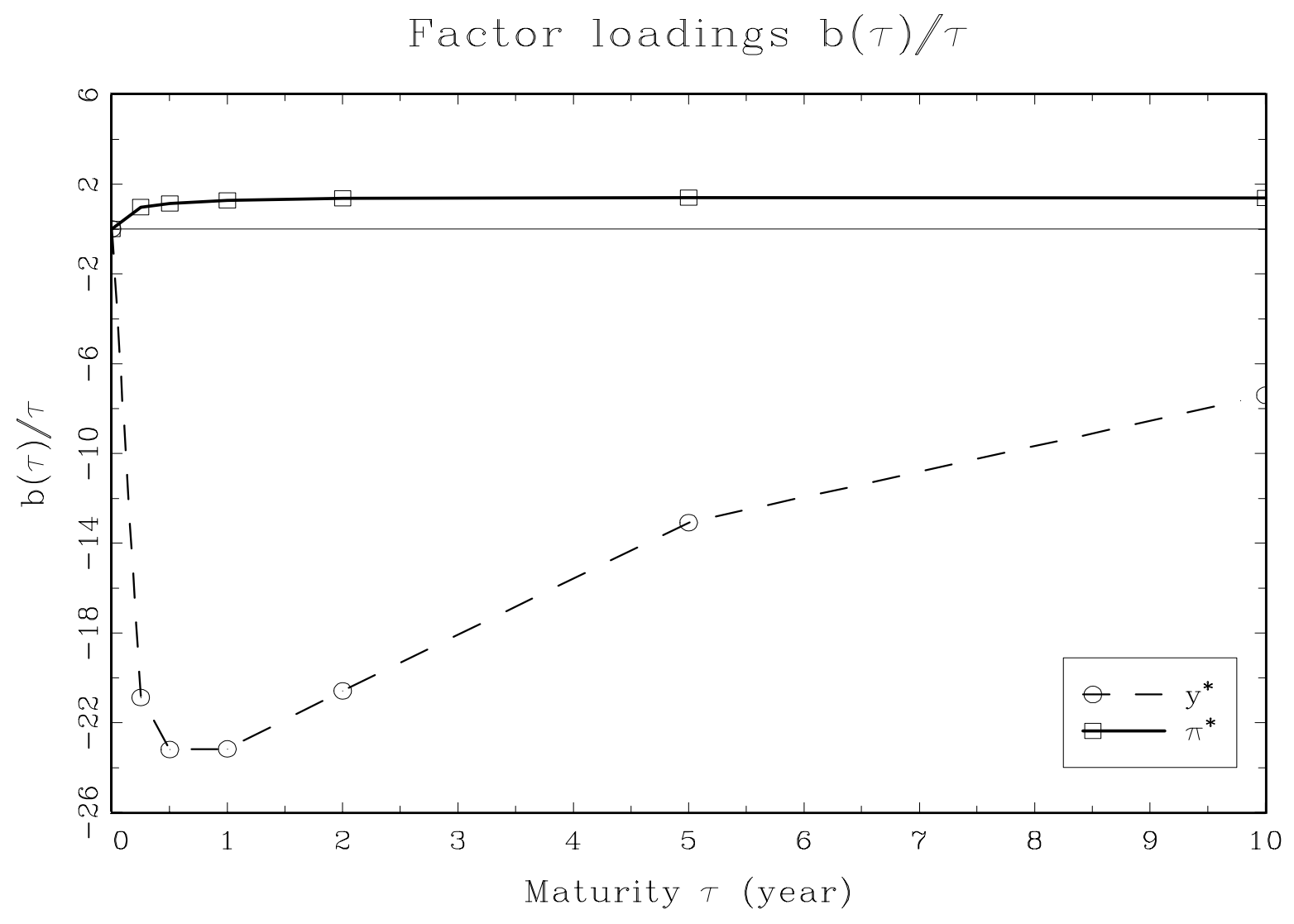

Figure B2: Estimated factor loadings - Factors 4 and 5 (1958-1979). 
Factor loadings $\mathrm{b}(\tau) / \tau$

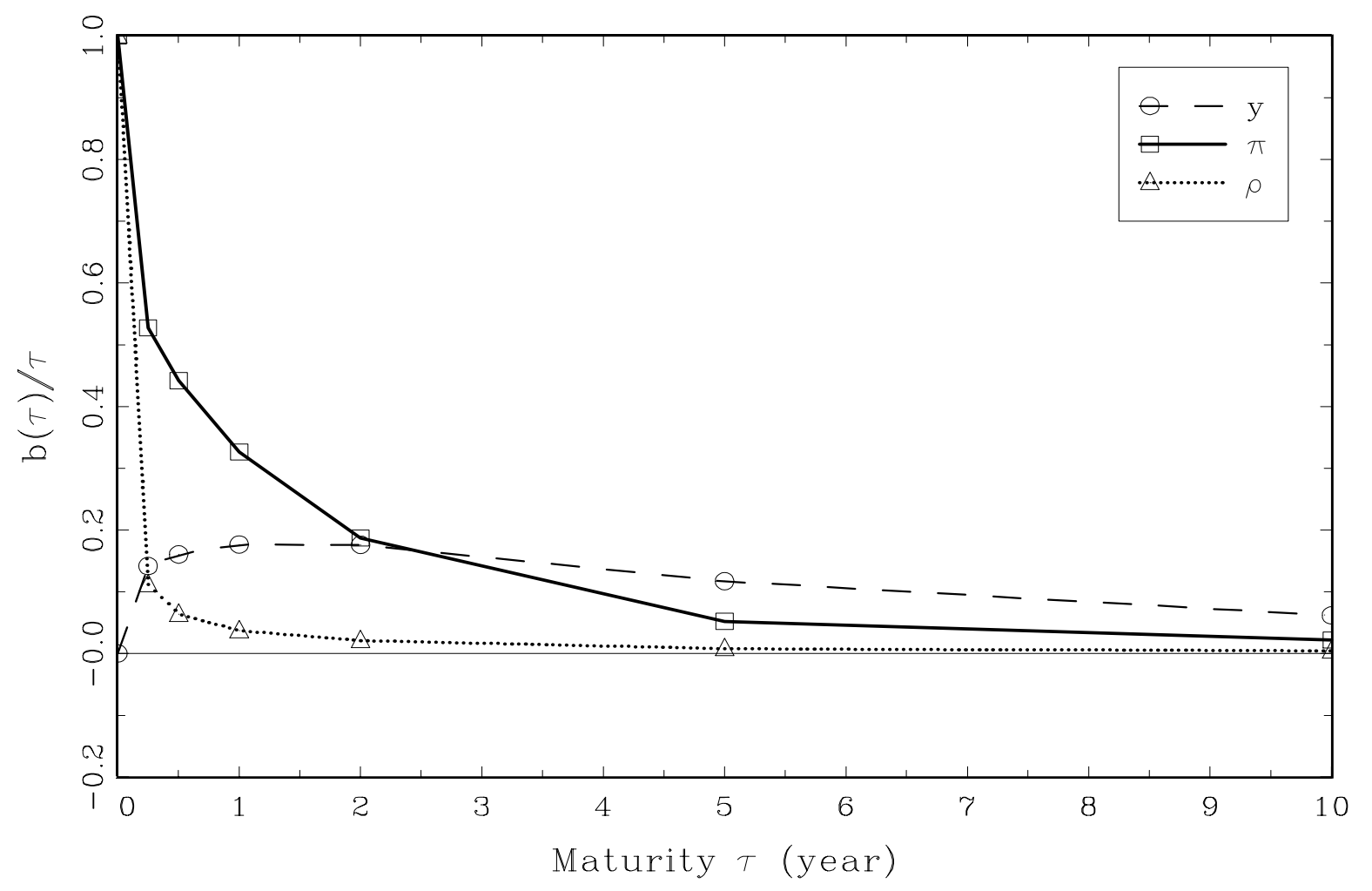

Figure B3: Estimated factor loadings - Factors 1 to 3 (1982-1998). 


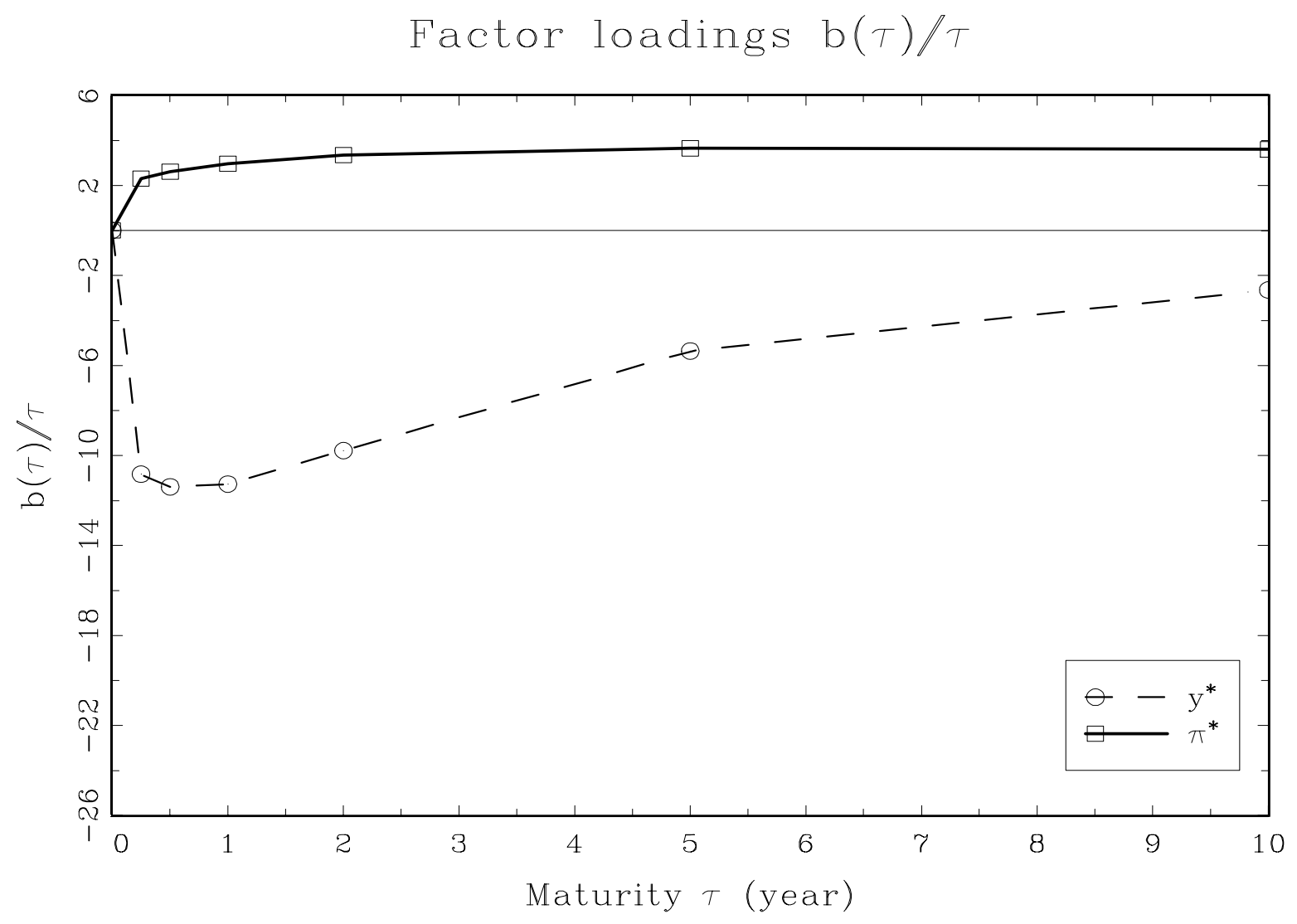

Figure B4: Estimated factor loadings - Factors 4 and 5 (1982-1998). 


\section{Appendix C: Inflation forecasting}

In this appendix, we briefly describe the computation of the inflation forecasts based on the model described in Section 2. For the one-year ahead inflation forecast, we use the following equation, with $h=1$ year:

$$
\pi_{f}^{1 y}=\pi^{*}+e^{\left(L_{\pi} h\right)}\left(\pi-\pi^{*}\right)
$$

where $L_{\pi}$ denotes the real part of the eigenvalue relative to inflation based on the $\mathbf{K}$ matrix (see also Section 3.1.2 and Table 3). For the computation of the forecasts for the average rate of inflation for the next ten years we have that:

$$
\bar{\pi}_{f}^{10 y}=\frac{1}{10} \sum_{h=1}^{10}\left[\pi^{*}+e^{\left(L_{\pi} h\right)}\left(\pi-\pi^{*}\right)\right]
$$

The results from an OLS regression of the inflation forecasts provided by the Survey of Professional Forecasters on the forecasts based on our model and a constant are reported in Table C1.

Table C1: Inflation forecast regressions

\begin{tabular}{ccc}
\hline \hline & 1 year ahead & average next 10 years \\
\hline \hline constant & 0.0004 & 0.0029 \\
& $(0.0015)$ & $(0.0023)$ \\
coefficient & 0.8898 & 0.8018 \\
& $(0.0289)$ & $(0.0474)$ \\
$\bar{R}^{2}$ & 0.89 & 0.84 \\
no.obs. & 116 & 55 \\
\hline \hline
\end{tabular}




\section{Figures}
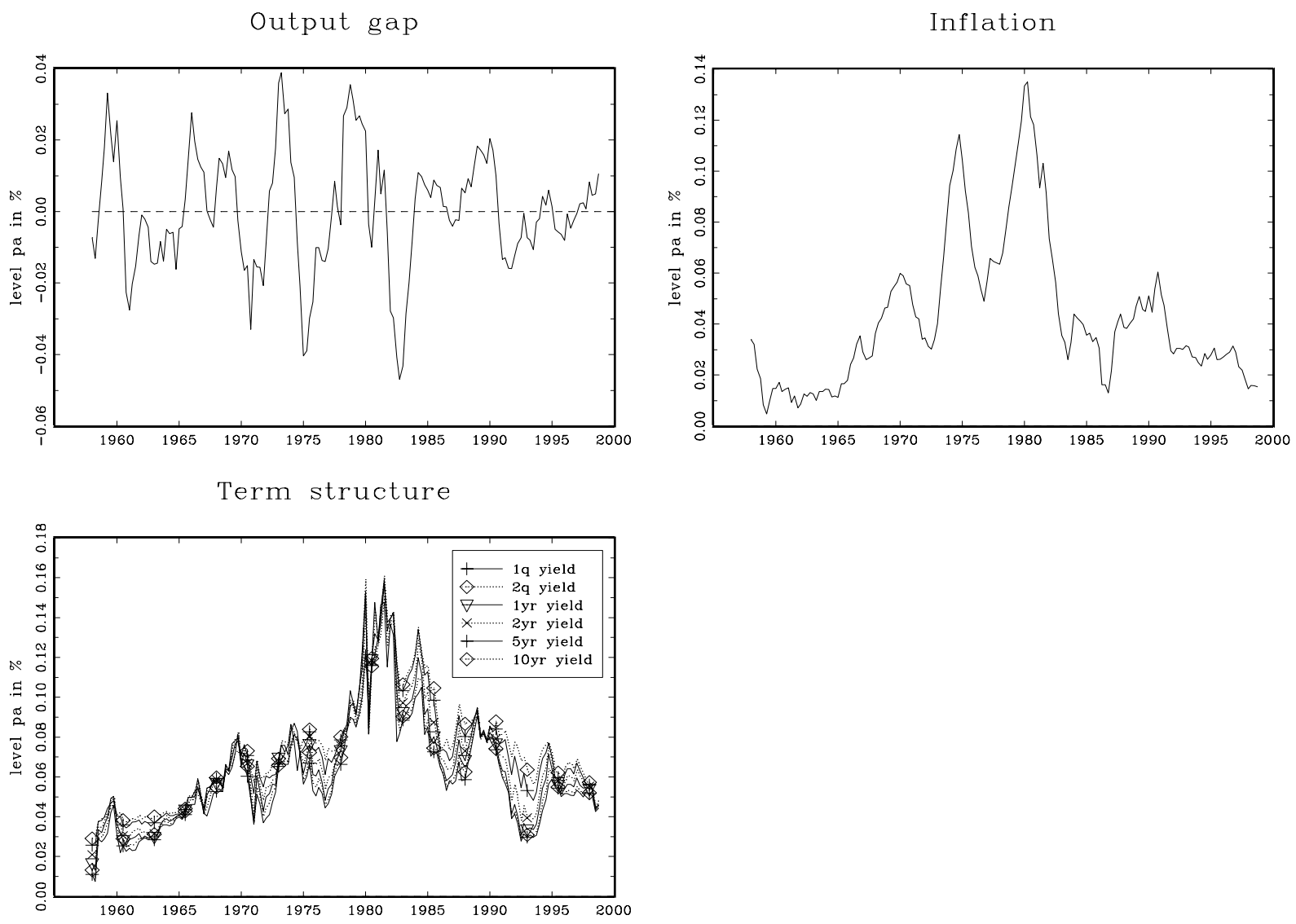

Figure 1: Data on output gap, inflation, and the term structure of interest rates (1958:Q1-1998:Q4). 

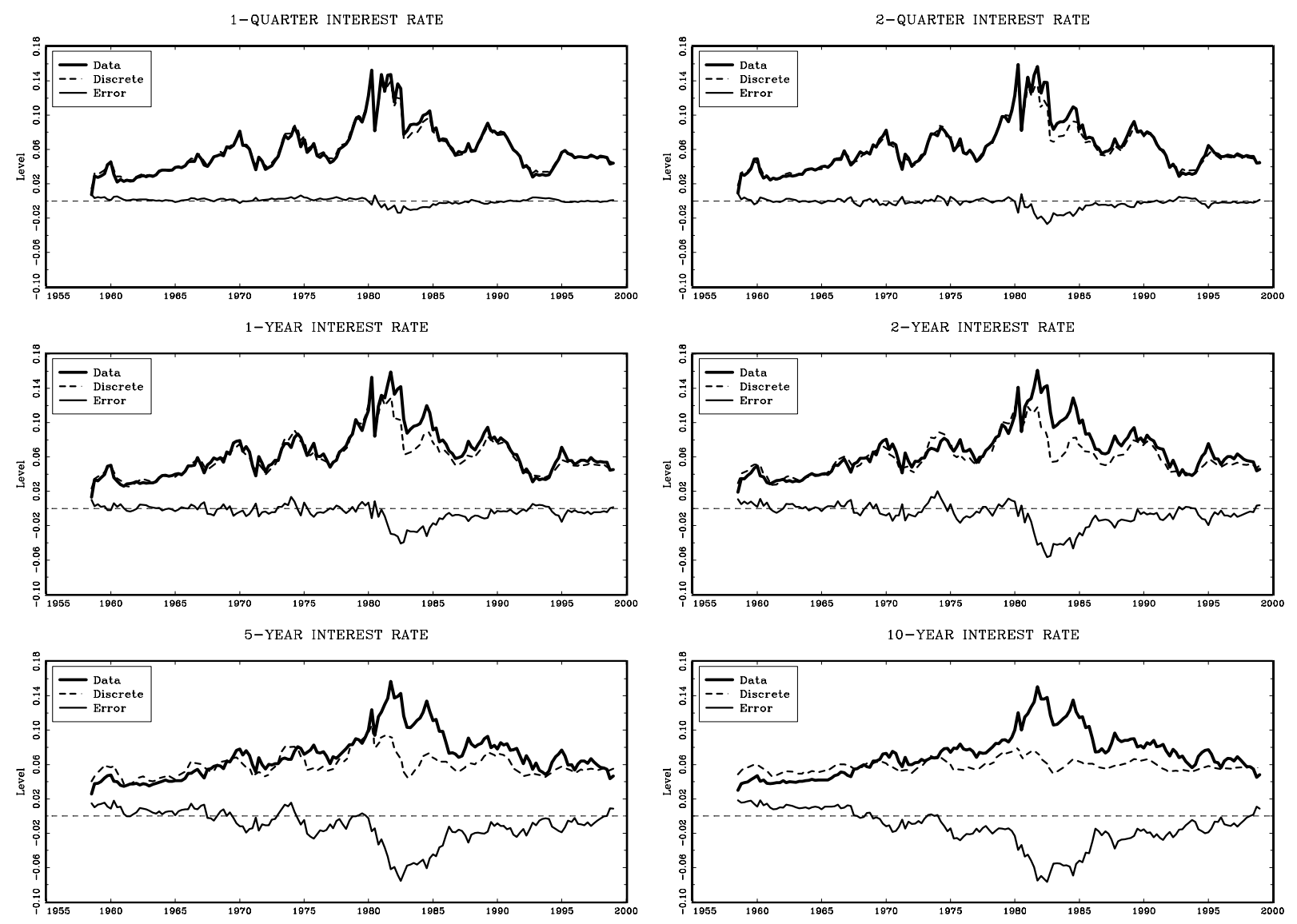

Figure 2: Term structure fit of discrete time model. 
OUTPUT
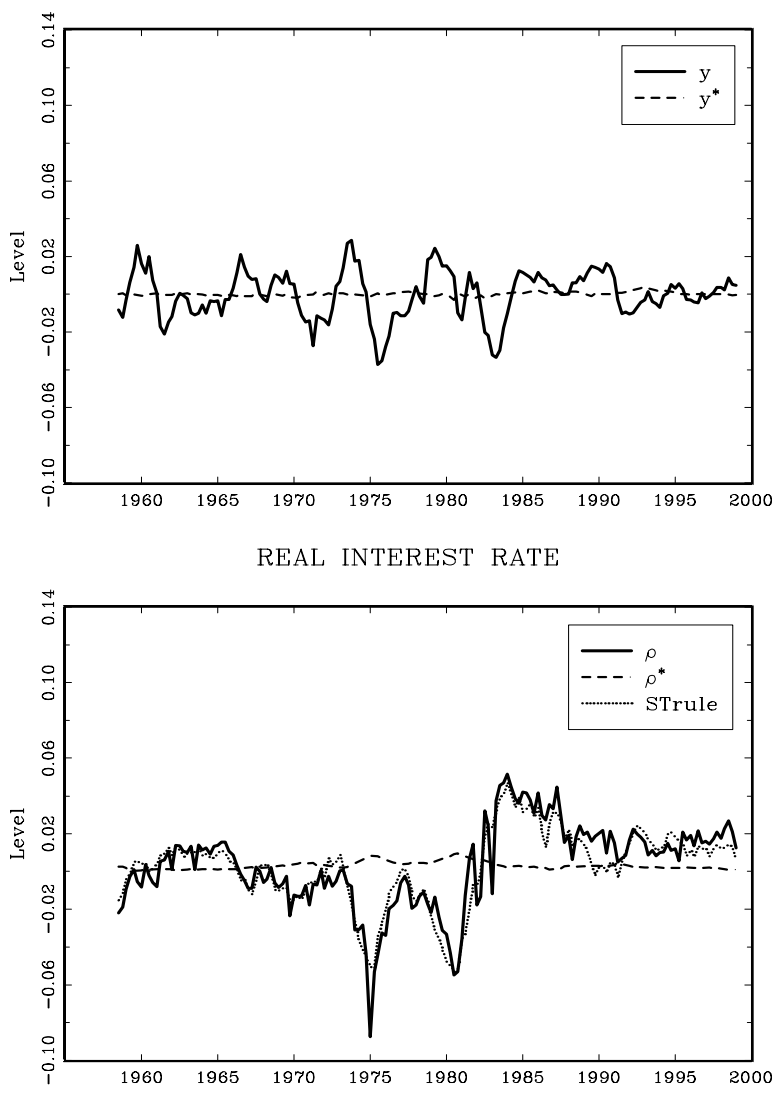

INFLATION

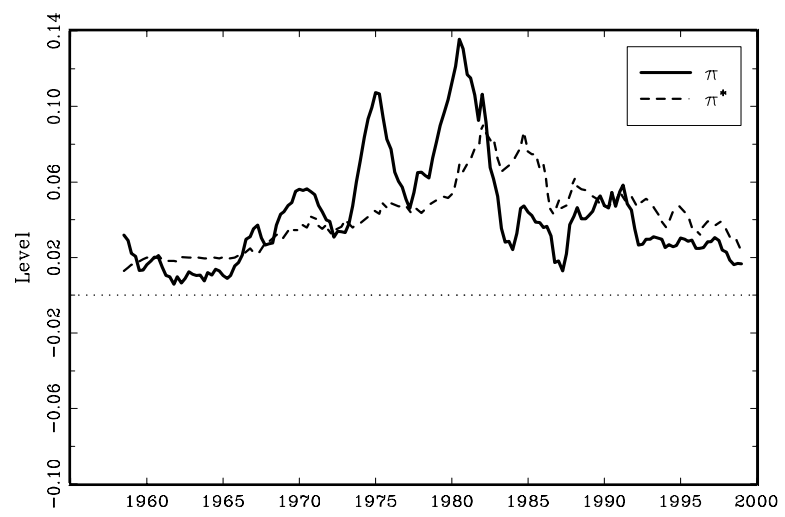

Figure 3: Macro-variables and their estimated central tendencies. 

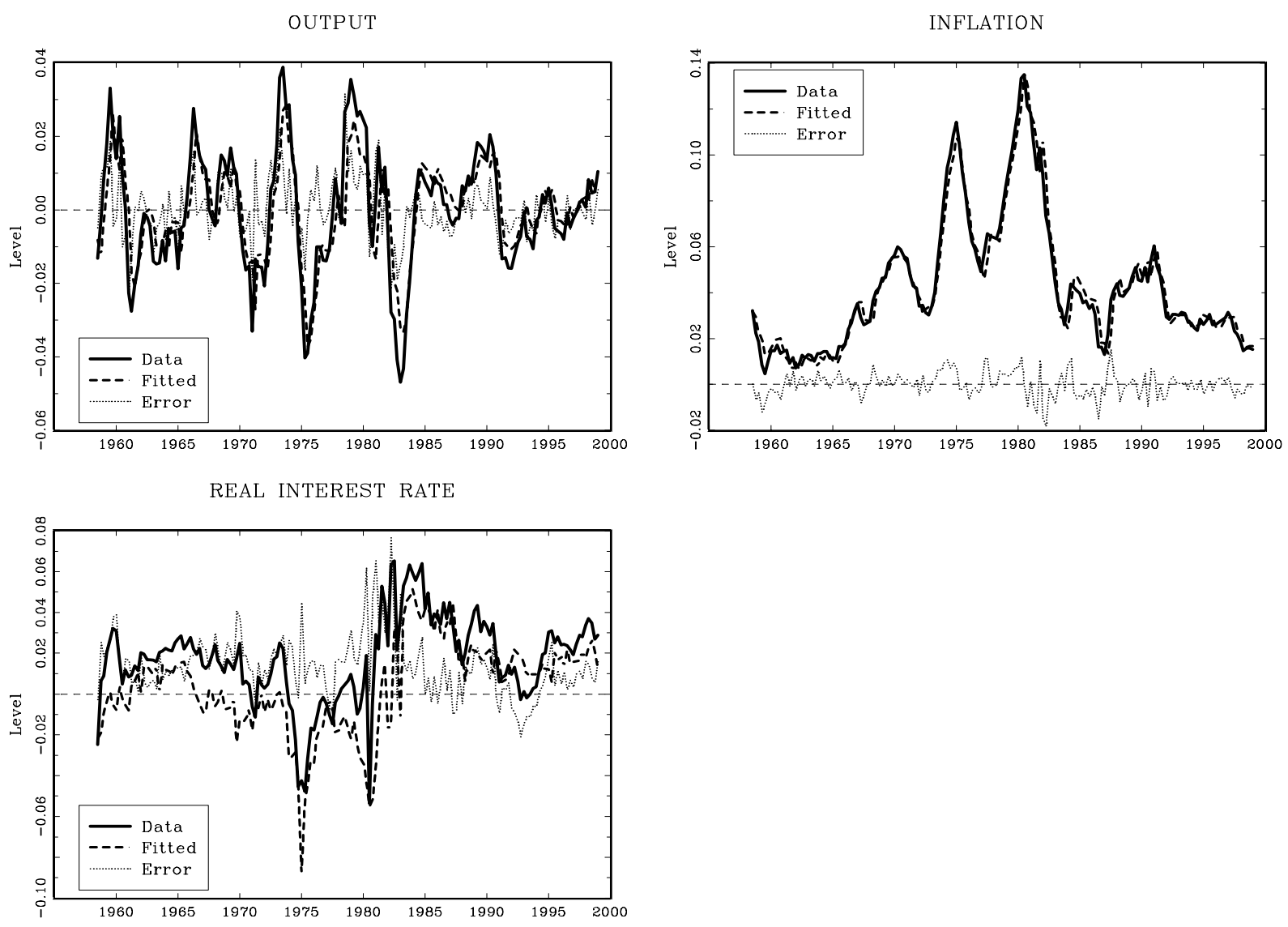

Figure 4: Model fit (model errors) of output gap, inflation, and real interest rate. 


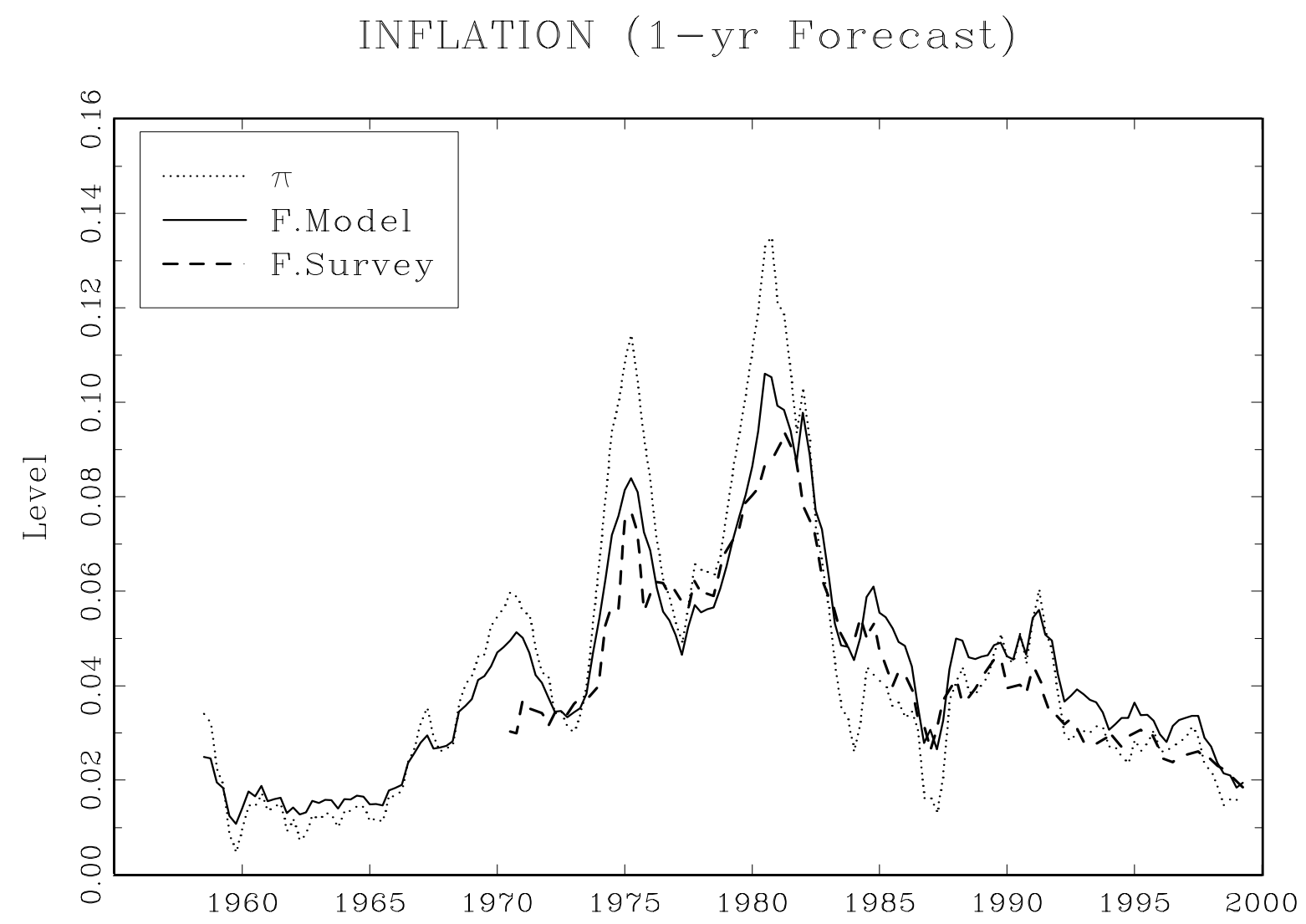

Figure 5: Comparison of average 1-year inflation forecast - Model vs. Survey of Professional Forecasters. 
INFLATION (10-yr Forecast)

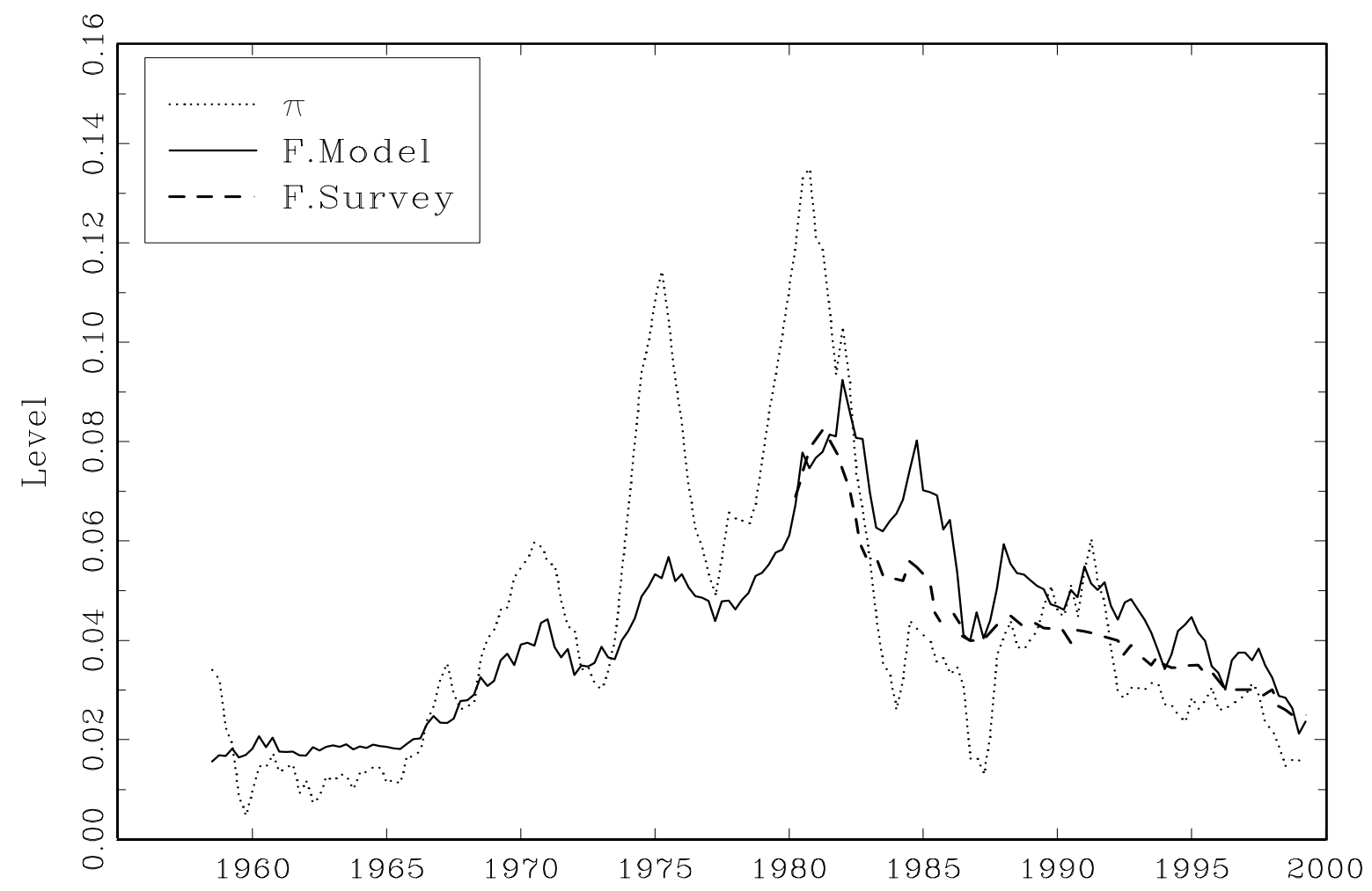

Figure 6: Comparison of average 10-year inflation forecast - Model vs. Survey of Professional Forecasters 
LEVEL EFFECT FACTOR

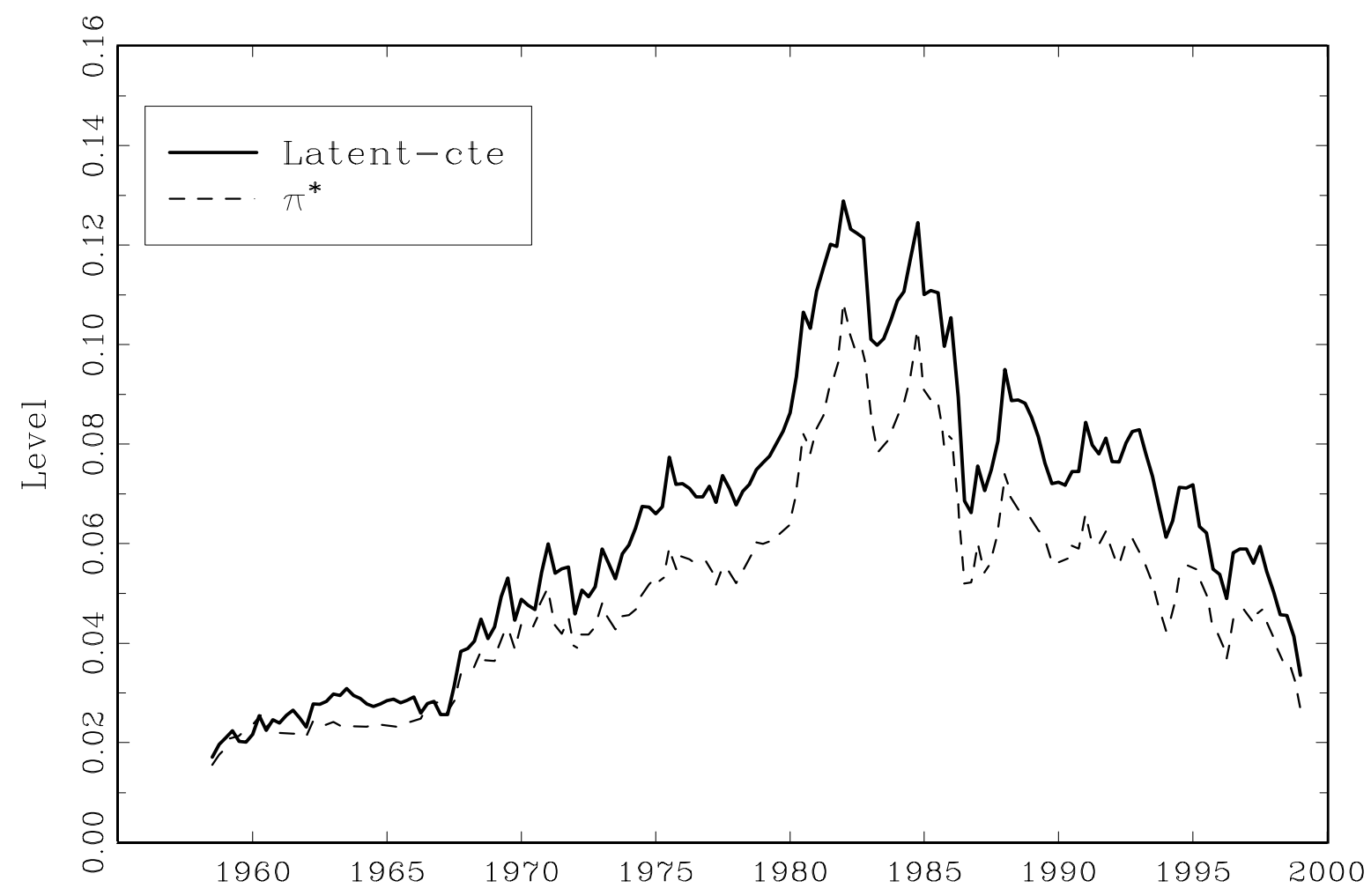

Figure 7: Interpretation of the Vasicek level factor. 

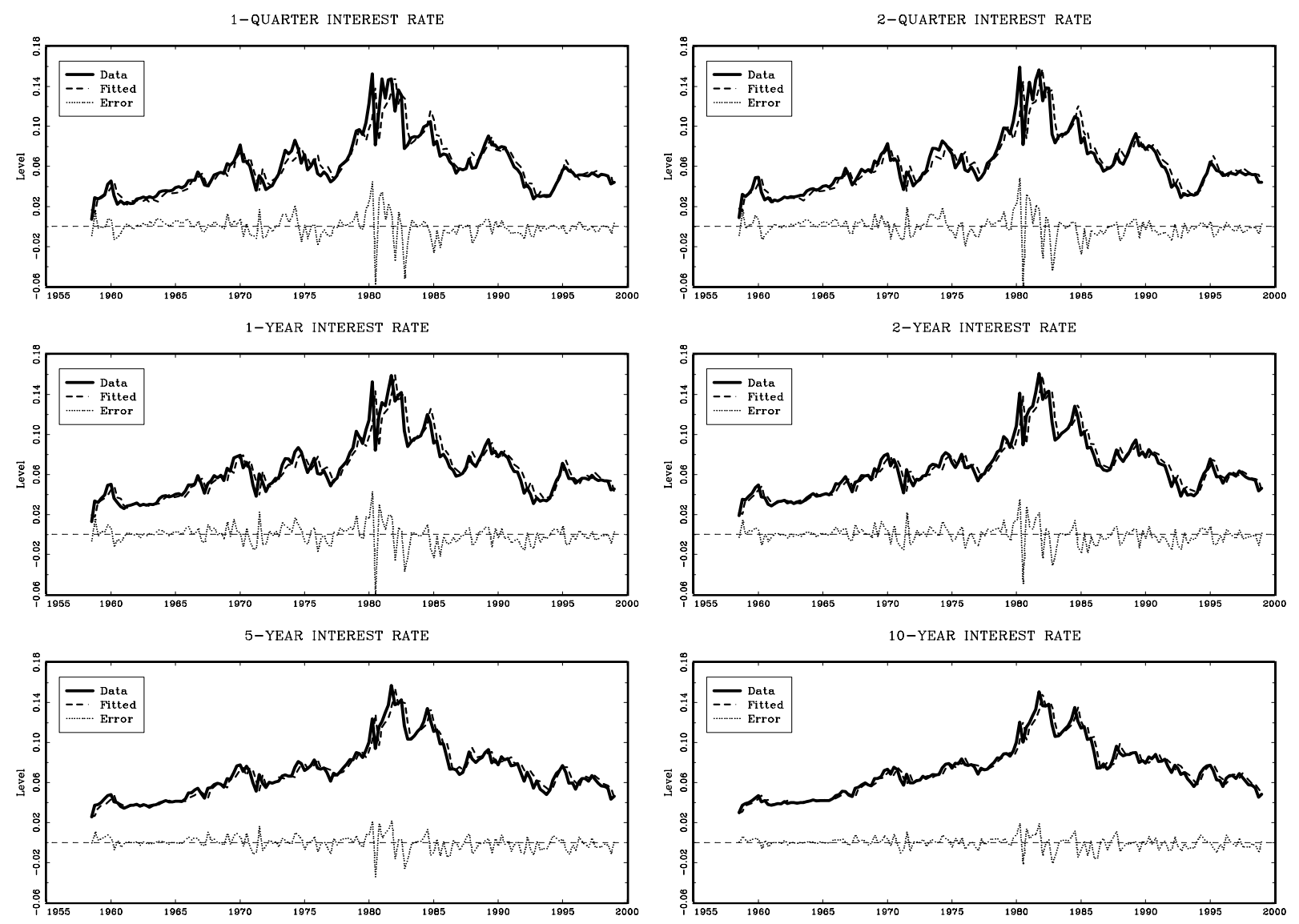

Figure 8: Model fit (model errors) of the term structure of interest rates. 


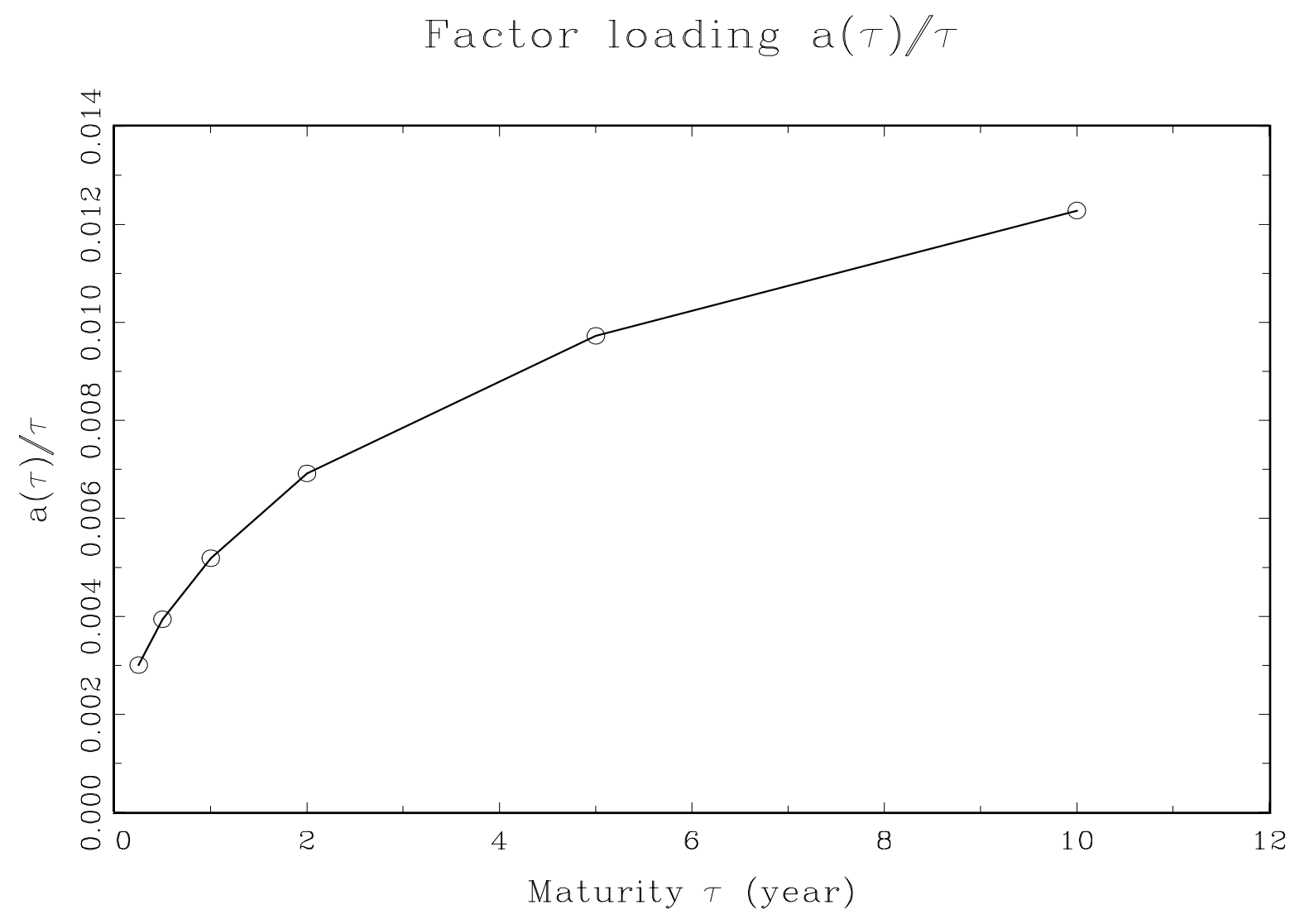

Figure 9: Estimated constant factor loading (1958-1998) 
Factor loadings $\mathrm{b}(\tau) / \tau$

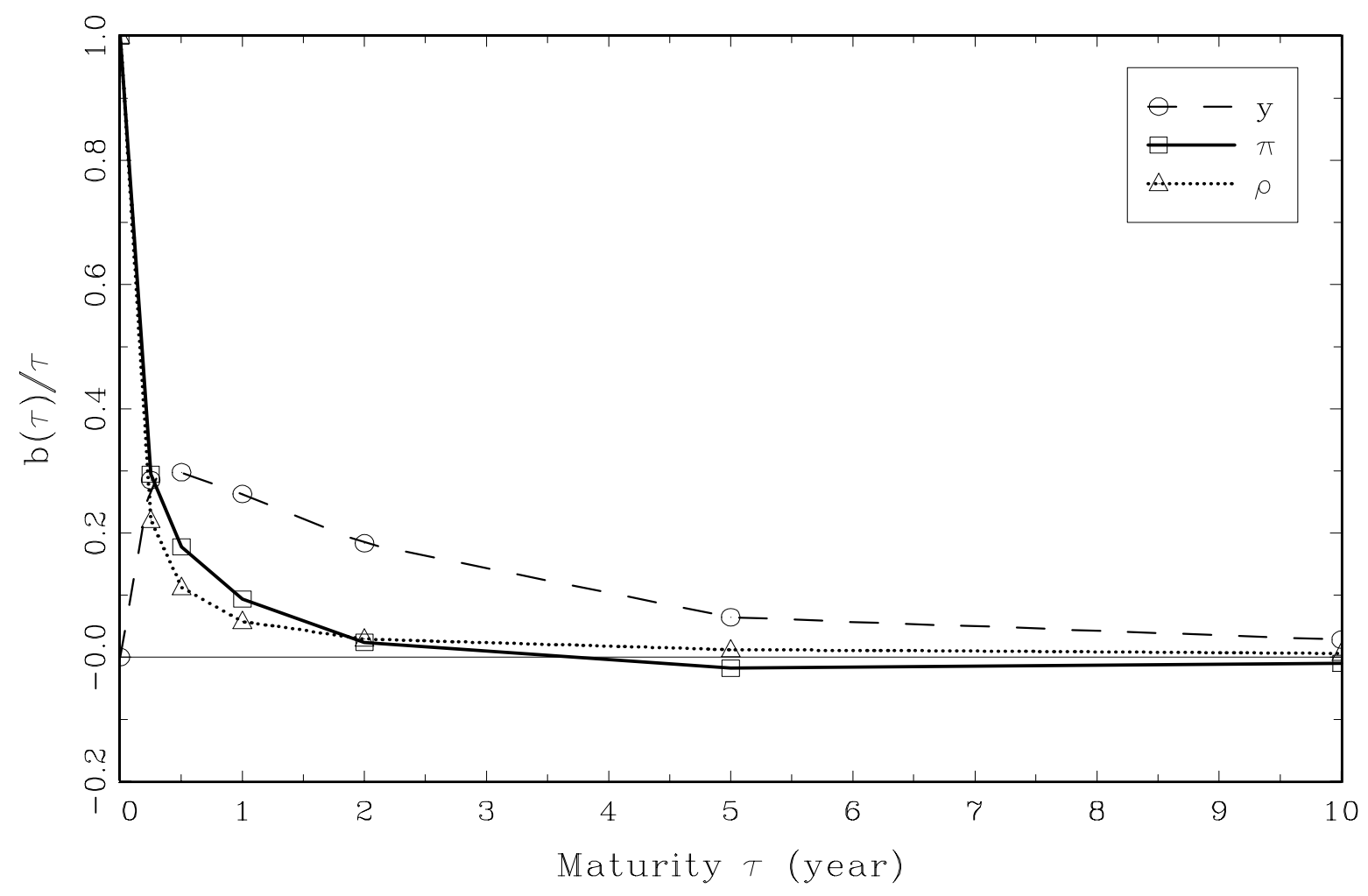

Figure 10: Estimated factor loadings - Factors 1 to 3 (1958-1998). 


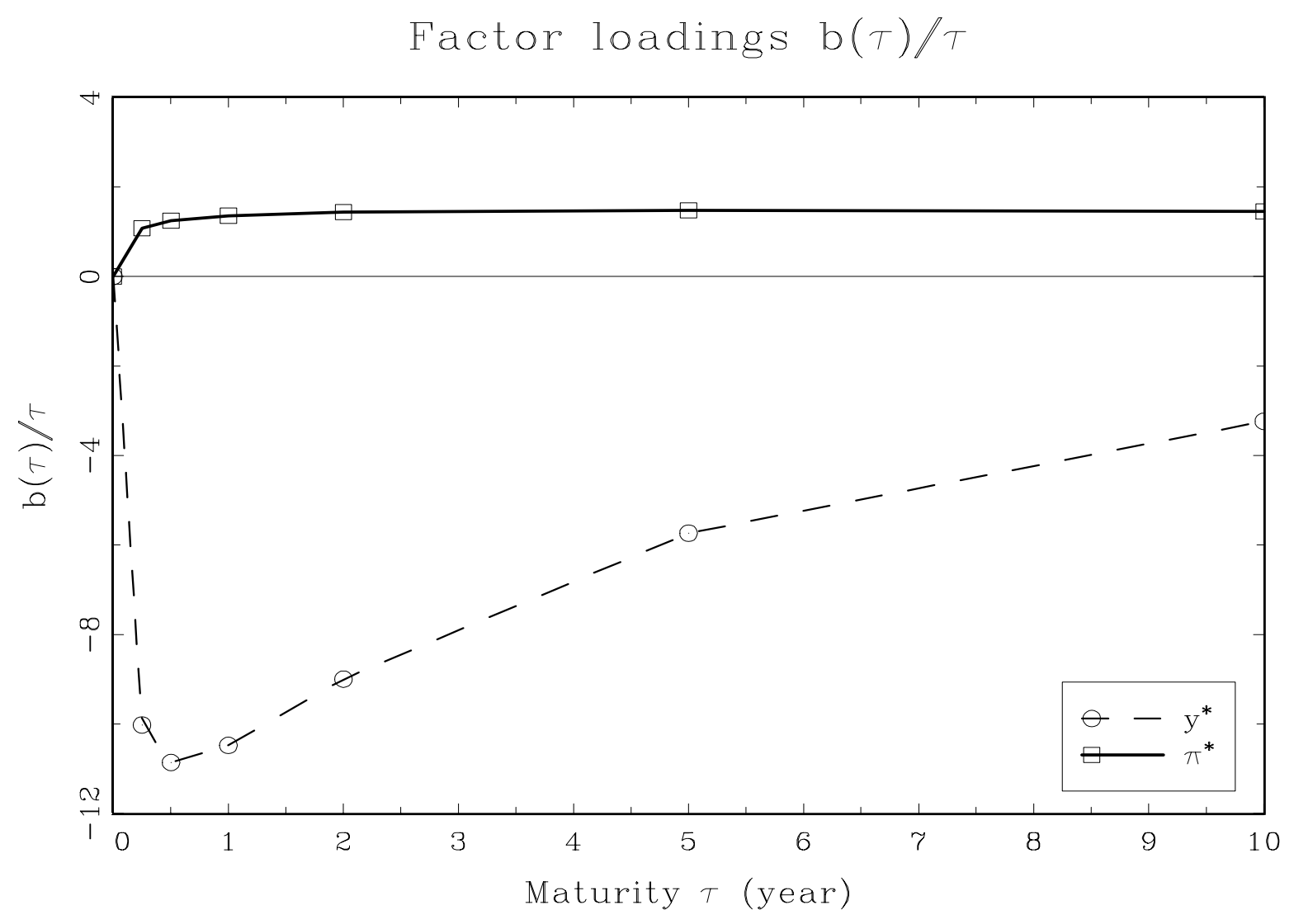

Figure 11: Estimated factor loadings - Factors 4 and 5 (1958-1998). 
CONSTANT

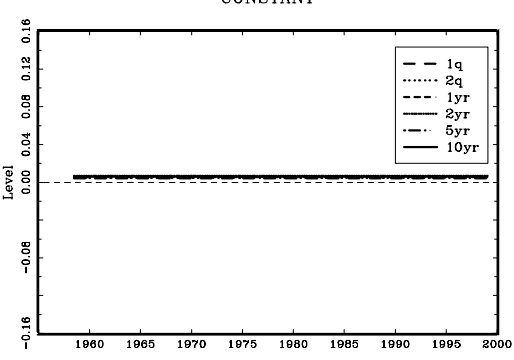

REAL INTEREST RATE
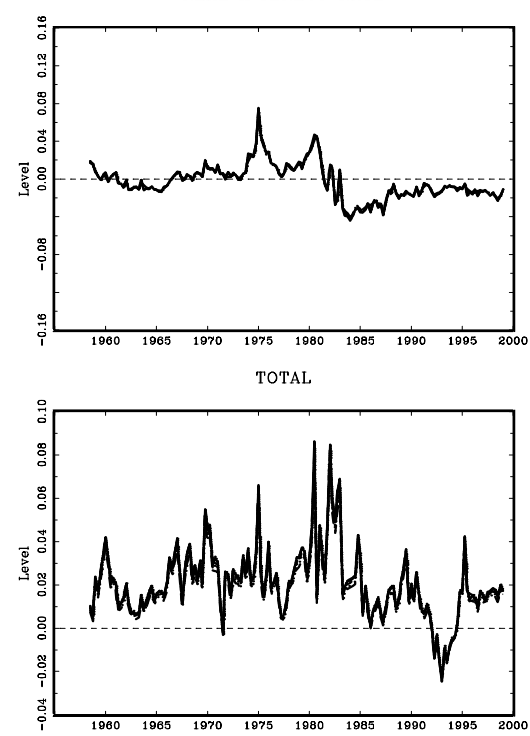

OUTPUT

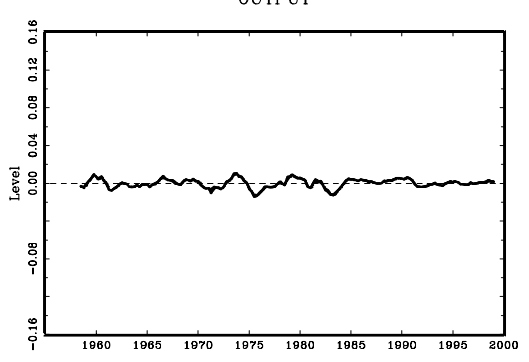

OUTPUT CENTRAL TENDENCY

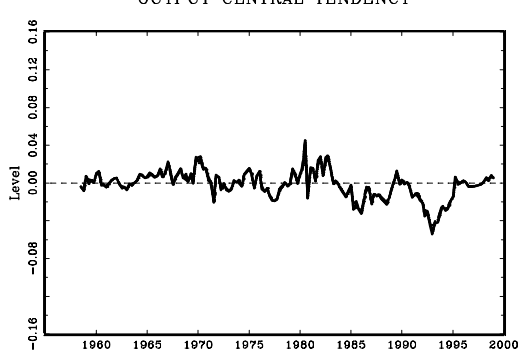

INFLATION

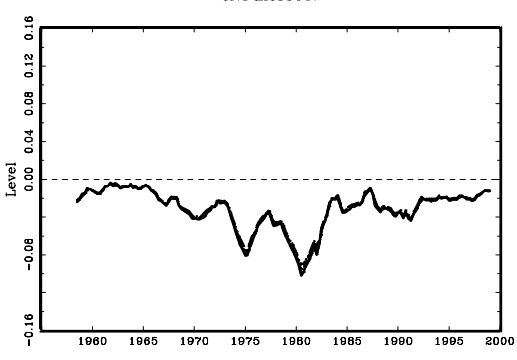

INFLATION CENTRAL TENDENCY

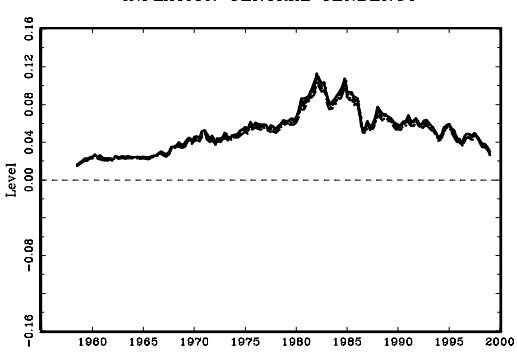

Figure 12: Factor decomposition of risk premium. 

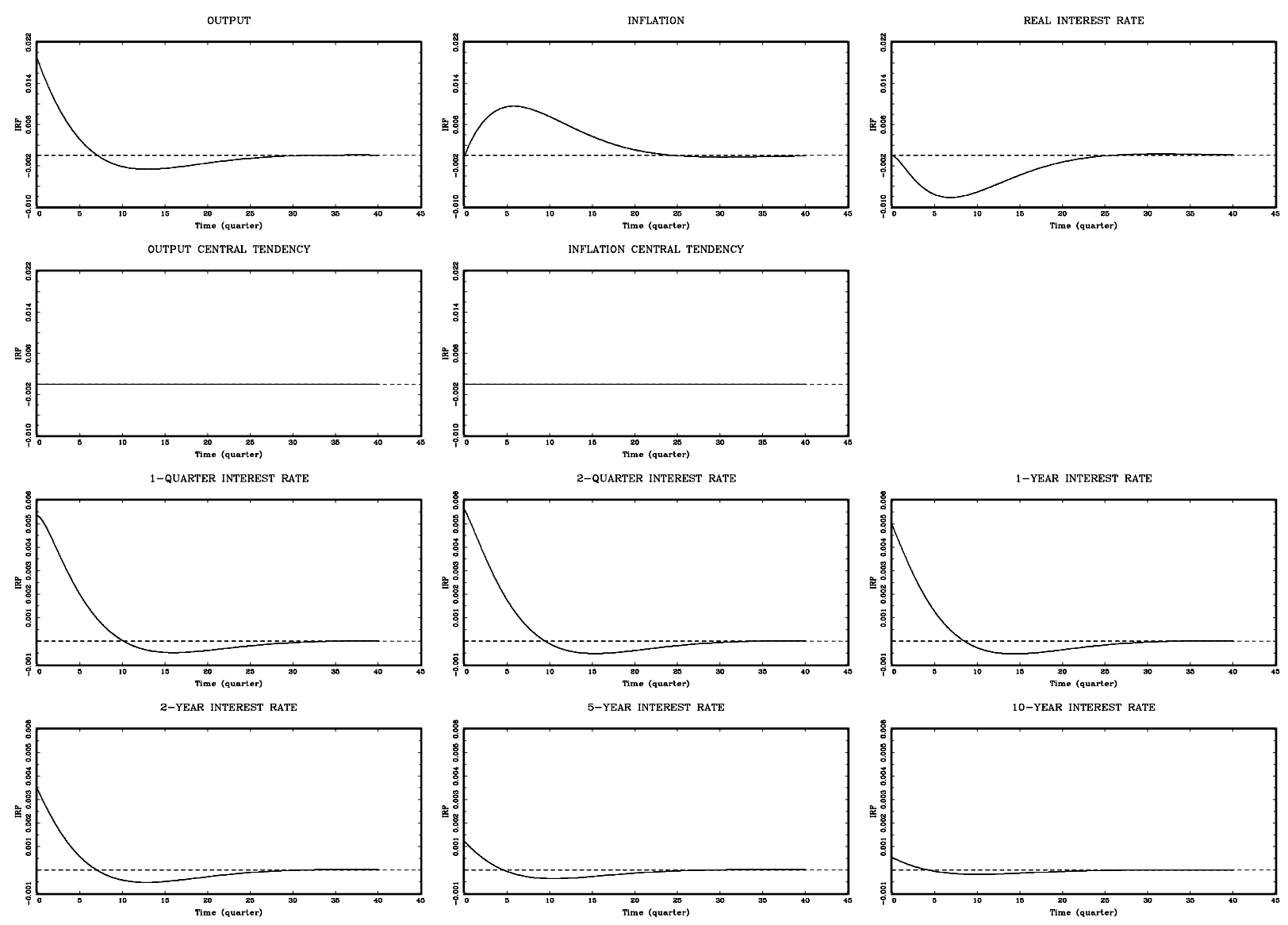

Figure 13: Impulse response analysis of output shocks. 

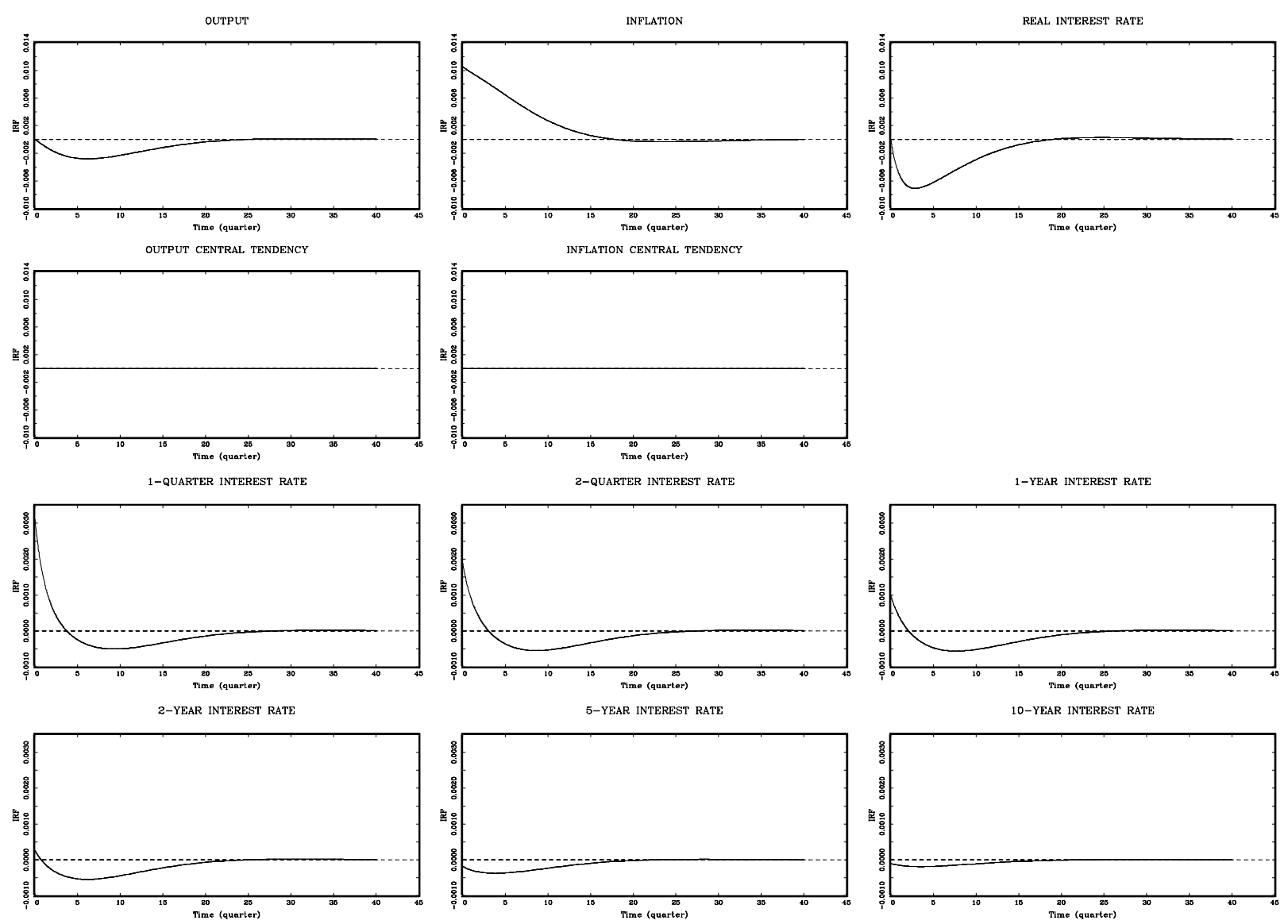

Figure 14: Impulse response analysis of inflation shocks. 

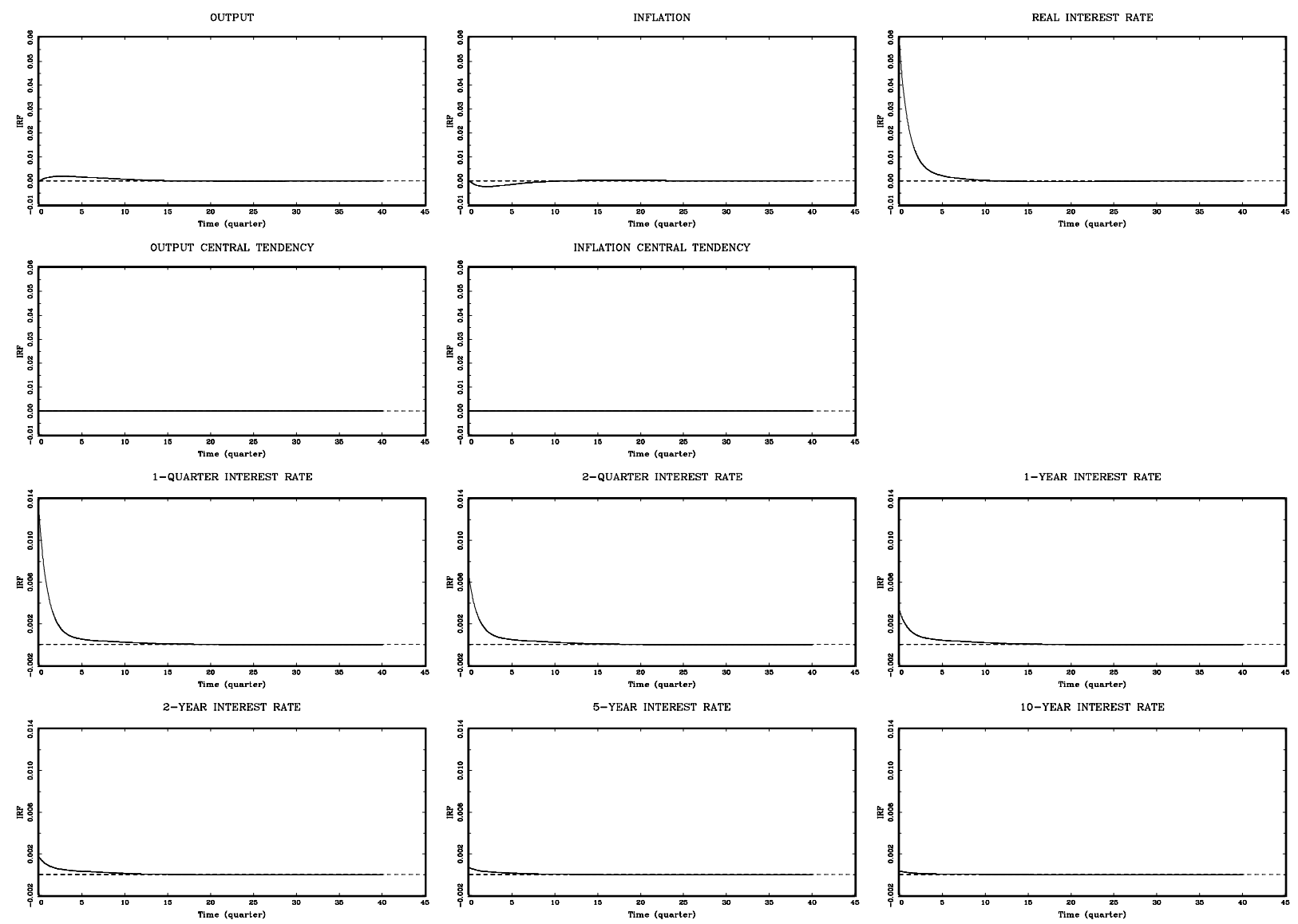

Figure 15: Impulse response analysis of real interest rate shocks. 
OUTPUT

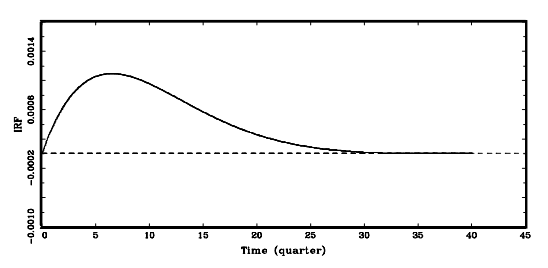

OUTPUT CENTRAL TENDENCY

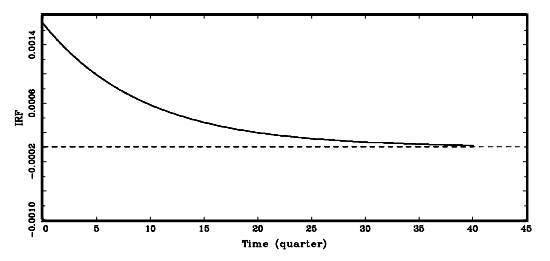

1-QUARTER INTEREST RATE
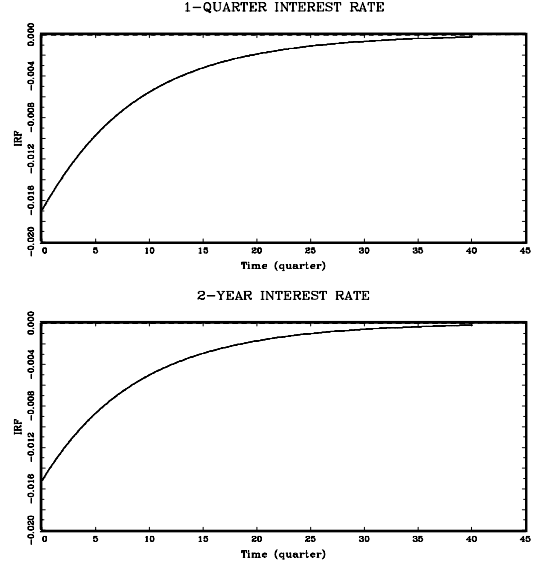

INFLATION

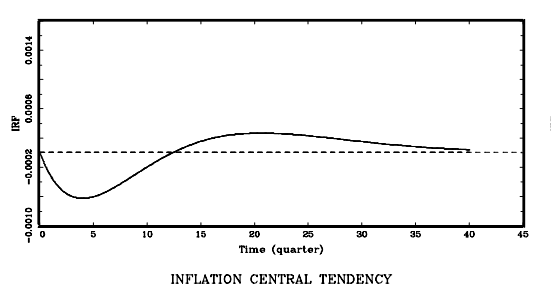

INFLATION CENTRAL TENDENCY

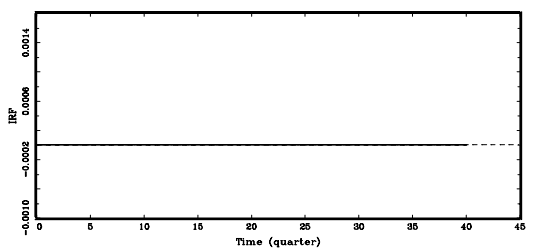

2-QUARTER INTEREST RATE
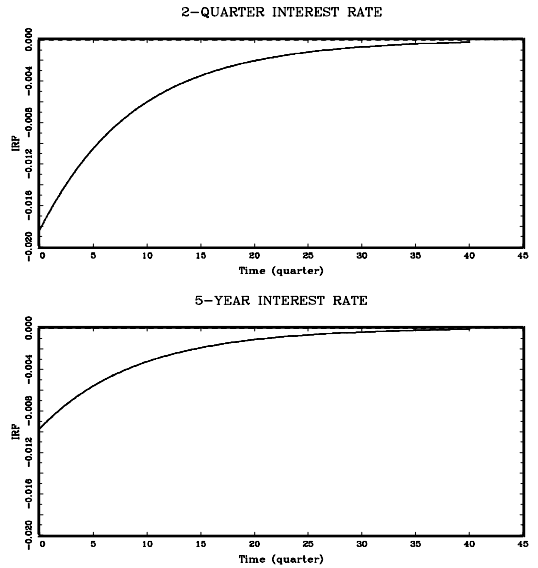

REAL INTEREST RATE
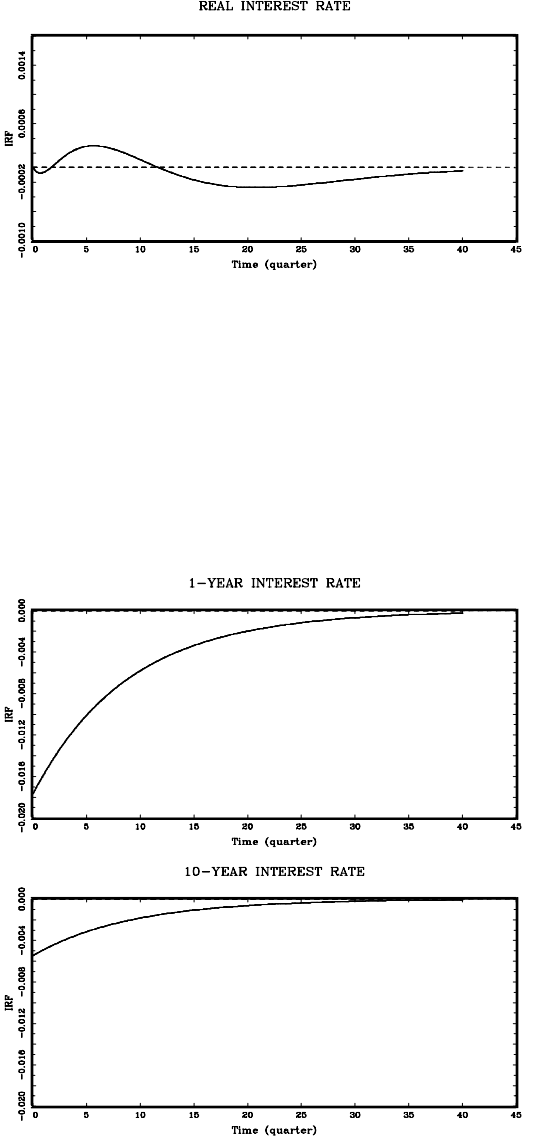

Figure 16: Impulse response analysis of shocks to the central tendency of output. 

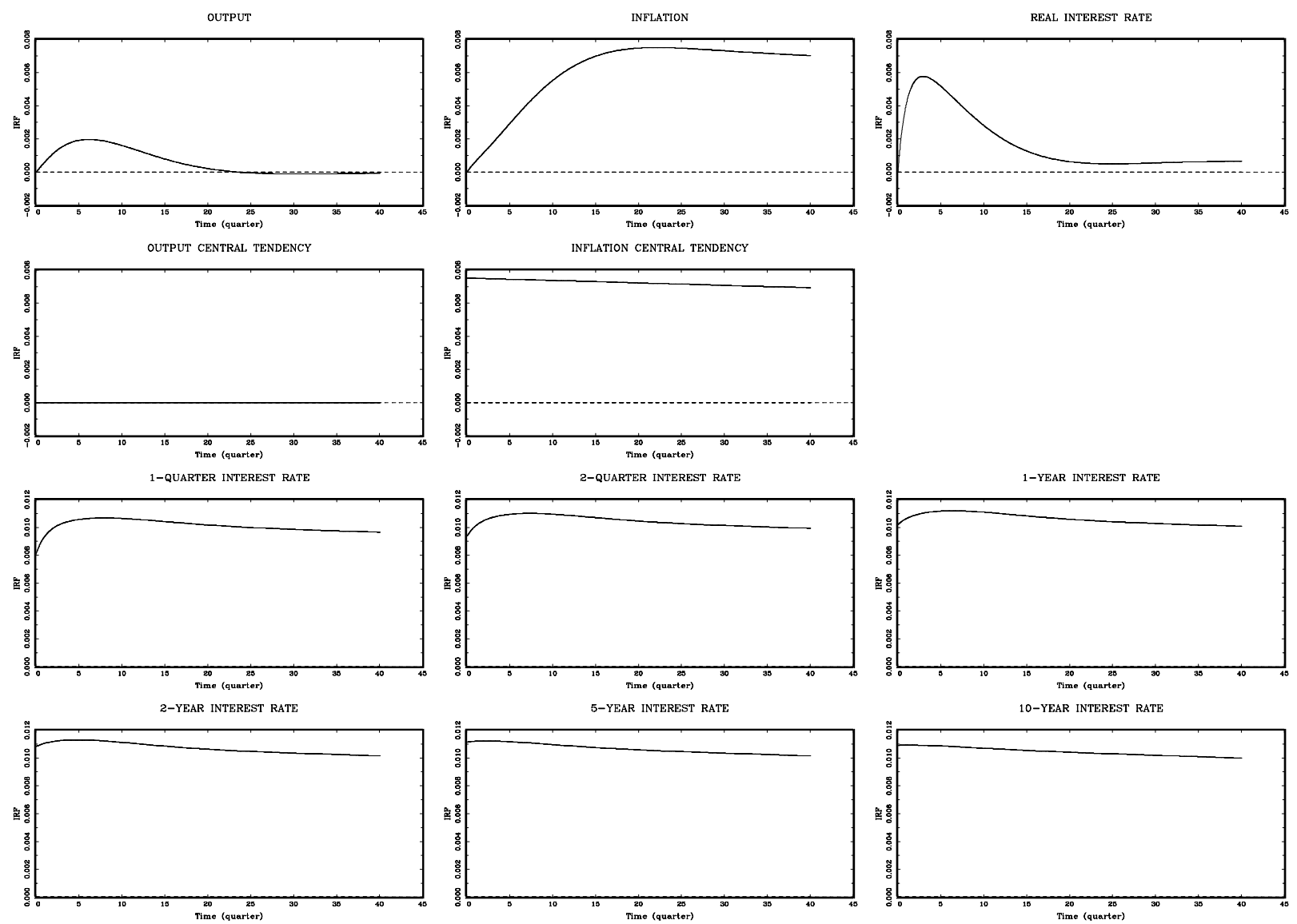

Figure 17: Impulse response analysis of shocks in the central tendency of inflation. 

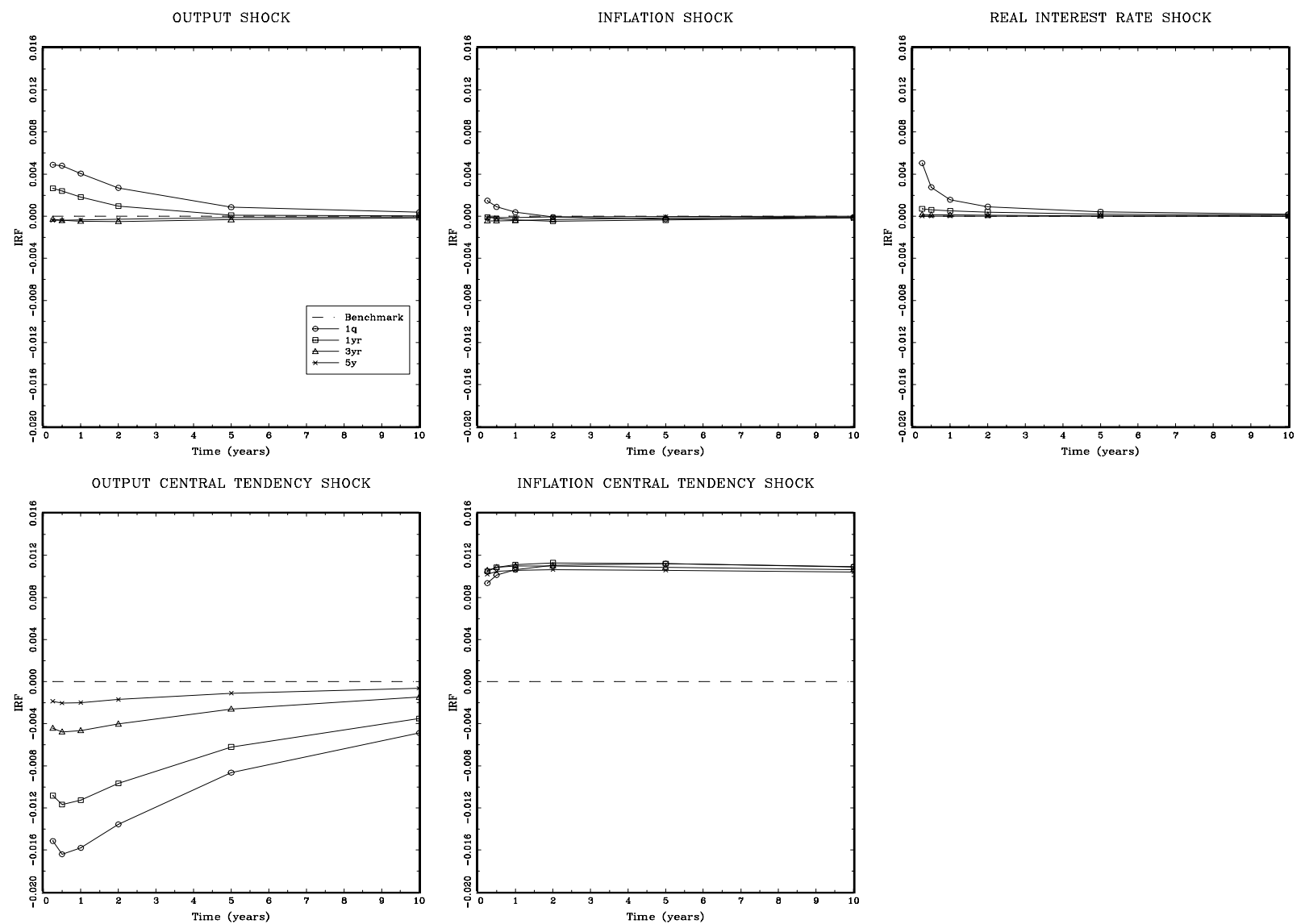

Figure 18: Time evolution of yield curve after a structural shock. 
OUTPUT PREDICTIONS vs. ALTERNATIVE

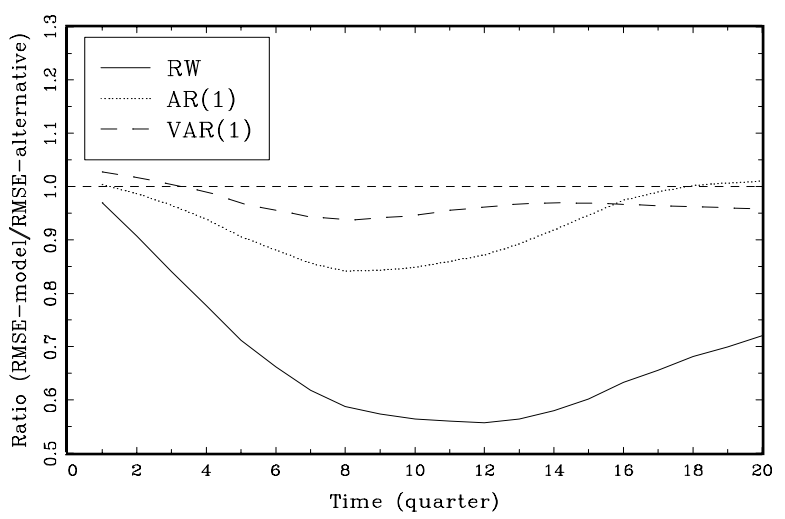

TERM STRUCTURE PREDICTIONS (Model vs. RW)

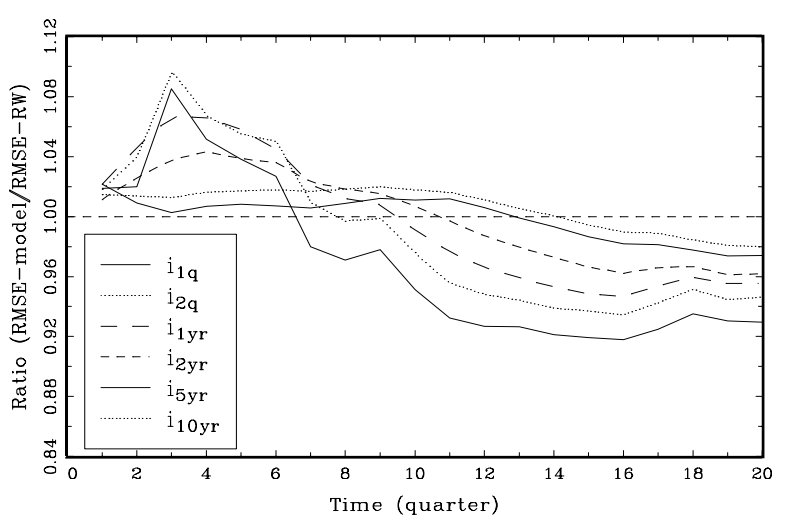

INFLATION PREDICTIONS VS. ALTERNATIVE

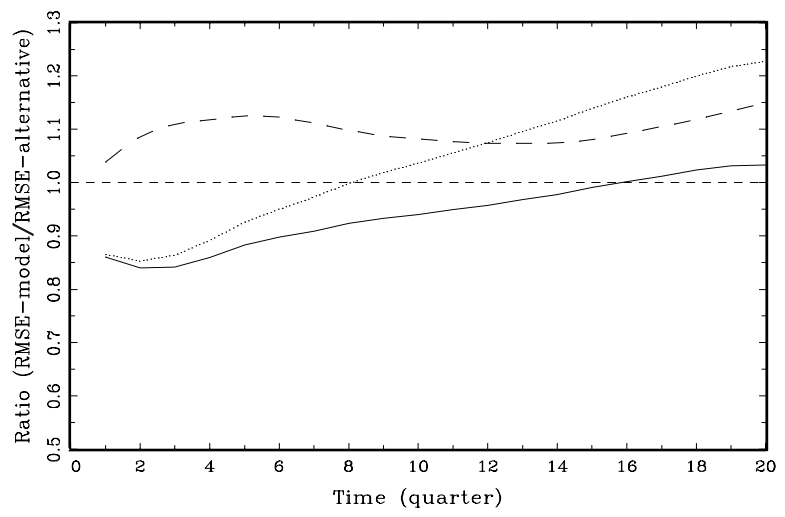

Figure 19: Forecasting performance of the model relative to the random walk, AR(1) and VAR(1). 
TERM STRUCTURE PREDICTIONS (Model vs. Latent)

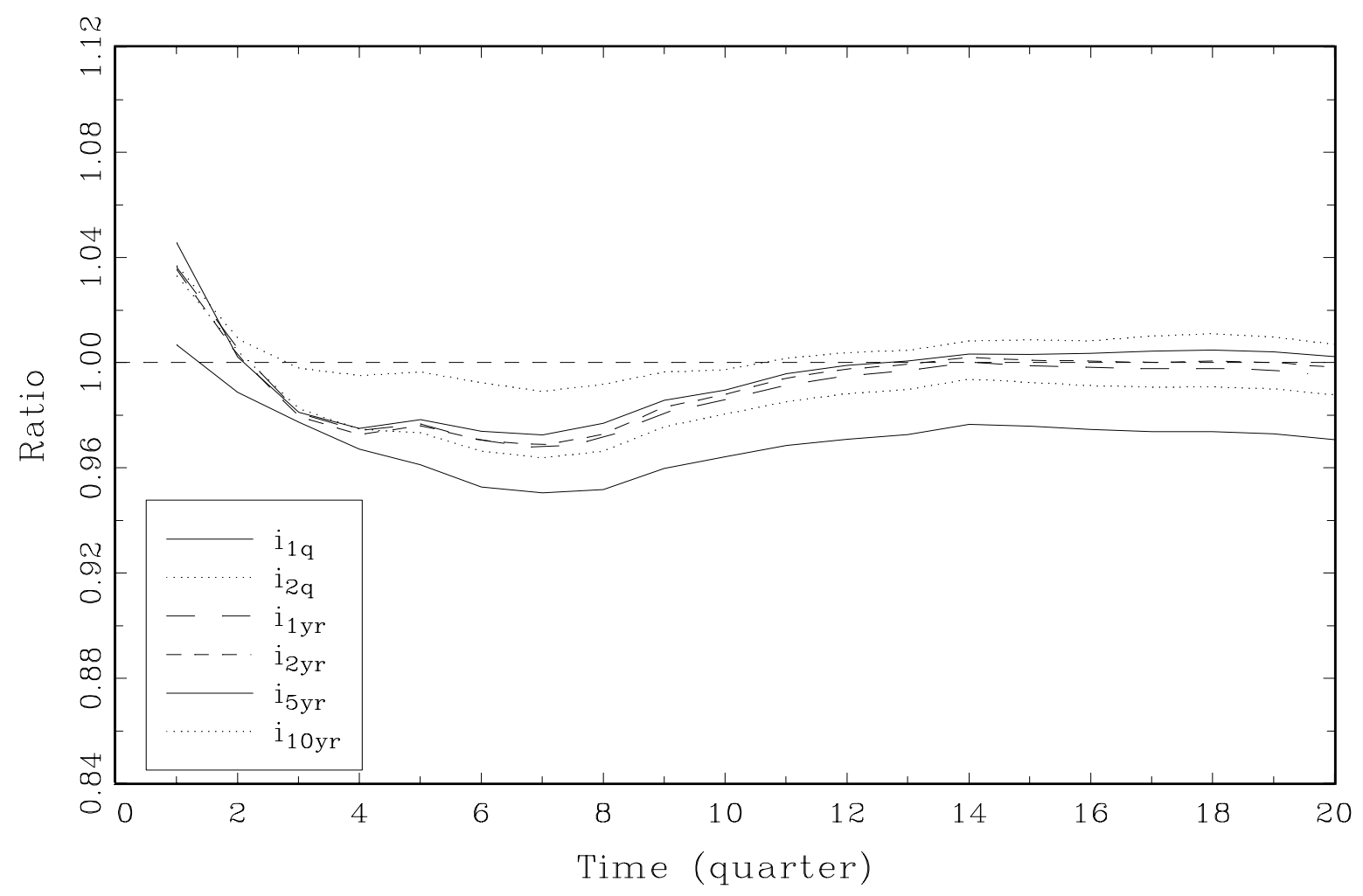

Figure 20: Forecasting performance of the model relative to the latent factor model. 